\title{
Grad's moment method for relativistic gas mixtures of Maxwellian particles
}

\author{
Gilberto M. Kremer* \\ Departamento de Física, Universidade Federal do Paraná, Curitiba, Brazil \\ Wilson Marques Jr. ${ }^{\dagger}$ \\ Departamento de Física, Universidade Federal do Paraná, Curitiba, Brazil
}

\begin{abstract}
Mixtures of relativistic gases are analyzed within the framework of Boltzmann equation by using Grad's moment method. A relativistic mixture of $r$ constituent is characterized by the moments of the distribution function: particle four-flows, energy-momentum tensors and third-order moment tensors. By using Eckart's decomposition and introducing $13 r+1$ scalar fields - related with the four-velocity, temperature of the mixture, particle number densities, diffusion fluxes, non-equilibrium pressures, heat fluxes and pressure deviator tensors - Grad's distribution functions are obtained. Grad's distribution functions are used to determine the third-order tensors and their production terms for mixtures whose constituent's rest masses are not too disparate, so that it follows a system of $13 r+1$ scalar field equations. By restricting to a binary mixture characterized by the six fields of partial particle number densities, four-velocity and temperature, the remainder 21 scalar equations are used to determine the constitutive equations for the non-equilibrium pressures, diffusion fluxes, pressure deviator tensors and heat fluxes. Hence the Navier-Stokes and generalized Fourier and Fick laws are obtained and the transport coefficients of bulk and shear viscosities, thermal conductivity, diffusion, thermal-diffusion and diffusion-thermal are determined. Analytic expressions for the transport coefficients in the non-relativistic and ultra-relativistic limiting case are given. Furthermore, solutions of the relativistic field equations for the binary mixture are obtained in form of forced and free waves. In the low frequency limiting case the phase velocity and the attenuation coefficient are determined for forced waves. In the small wavenumber limiting case it is shown that there exist four longitudinal eigenmodes, two of them corresponding to propagating sound modes and two associated with non-propagating diffusive modes.
\end{abstract}

PACS numbers: 51.10.+y, 05.20.Dd, 47.75.+f

\footnotetext{
*kremer@fisica.ufpr.br

$\dagger$ marques@fisica.ufpr.br
} 


\section{INTRODUCTION}

We may state that the beginning of the relativistic kinetic theory goes back to 1911 when Jüttner [1] derived an equilibrium distribution function for a relativistic gas, which in the non-relativistic limiting case becomes the Maxwellian distribution function. Jüttner has also succeeded to derive in 1928 [2] the relativistic Bose-Einstein and Fermi-Dirac distribution functions. The covariant formulation of the Boltzmann equation was proposed by Lichnerowicz and Marrot [3] in the forties of the last century and in the sixties the determination of the transport coefficients from the Boltzmann equation by using the Chapman-Enskog methodology was obtained by Israel [4] and Kelly [5].

Mixtures of relativistic gases are important in the field of astrophysics, in particular to problems associated with gases at high temperature in a stellar interior. Within the framework of Boltzmann equation these mixtures were studied by several authors and among others we quote the works [6-18]. In these works the usual methodology applied to determine the transport coefficients was the Chapman-Enskog method and general expressions for the transport coefficients were determined for generic differential cross sections. Only few explicit expressions for the transport coefficients were determined for very special cases of binary mixtures of Maxwellian particles [8] and hard spheres [13] where the constituents have the same rest masses or a Lorentz gas of hard spheres [14].

In this work we analyze mixtures of relativistic gases of Maxwellian particles by using Grad's moment method applied to the system of Boltzmann equations. In relativistic kinetic theory there is no unique differential cross section which tends in the non-relativistic limiting case to the one which is proportional to the relative velocity, known as the differential cross section of Maxwellian particles. At least three of them given in the works [4, 19, 20] have this property, and here we shall use the one defined in [20].

We are interested in a mixture that is characterized by $r$ constituents and described by $13 r+1$ basic fields of fourvelocity, temperature of the mixture, particle number densities, diffusion fluxes, non-equilibrium pressures, heat fluxes and pressure deviator tensors. Grad's distribution functions are obtained from the definition of the basic fields which are related with the Eckart decomposition of the particle four-flows and of the energy-momentum tensors. From the knowledge of Grad's distribution functions the third-order moments of the distribution functions are calculated and the production terms that appear in the balance equations are determined for the case of a mixture of Maxwellian particles for constituents whose rest masses are not too disparate. Once the $13 r+1$ field equations for the basic fields are established, the mixture is restricted to a binary mixture characterized by six fields of particle number densities, four-velocity and temperature of the mixture. The constitutive equations for the diffusion flux, pressure deviator tensors, non-equilibrium tensors and heat fluxes are determined by a method akin to the Maxwellian iteration procedure applied to the remaining 21 scalar field equations. The generalized laws of Fick, Fourier and Navier-Stokes are obtained and the coefficients of diffusion, thermal-diffusion, diffusion-thermal, thermal conductivity and bulk and shear viscosities are determined. Explicit expressions for these coefficients in the non-relativistic and ultra-relativistic limiting cases are given, as well as their graphics as functions of a parameter which represents the ratio of the rest energy of a particle and the thermal energy of the gas. From the system of field equations for the binary mixture we have analyzed the solutions corresponding to small perturbations from an equilibrium state related with the propagation of forced and free waves. For the acoustic solution in the low frequency limiting case we have determined the phase velocity and the attenuation coefficient. For the eigenmodes in the small wavenumber limiting case we have shown the occurrence of two propagating sound modes and two non-propagating diffusive modes.

This work is structured as follows: in Section II the Boltzmann equations, the moments of the distribution functions and their balance equations are introduced. The Eckart decomposition is the subject of Section III and Grad distribution functions are determined in Section IV. In Section V the constitutive equations for the third-order moment tensors and for the production terms are obtained and in Section VI the linearized $13 r+1$ field equations are established. A binary mixture with six scalar fields of particle number densities, four-velocity and temperature of the mixture is analyzed in Section VII, where the generalized laws of Fick, Fourier and Navier-Stokes are obtained from a method akin to the Maxwellian iteration procedure. Furthermore, the transport coefficients of diffusion, thermal-diffusion, diffusion-thermal, thermal conductivity and bulk and shear viscosities associated with these laws are determined in the non-relativistic and ultra-relativistic limiting cases. In Section VIII solutions of the binary mixture field equations are analyzed in terms of sound propagation and eigenmodes. Finally, the main conclusions of this work are discussed in Section IX.

Latin indexes running from $a=1 \ldots r$ specify the constituents of the mixture, while Greek indexes running from $\alpha=0,1,2,3$ denote the space-time coordinates. 


\section{BOLTZMANN AND TRANSFER EQUATIONS}

Let us consider a relativistic gas mixture of $r$ constituents in a Minkowski space with metric tensor $\left(\eta_{\alpha \beta}\right)=$ $\operatorname{diag}(1,-1,-1,-1)$. The particles of constituent $a=1, \ldots r$ have rest mass $m_{a}$ and are characterized by the spacetime coordinates $\left(x^{\alpha}\right)=(c t, \mathbf{x})$ and momenta $\left(p_{a}^{\alpha}\right)=\left(p_{a}^{0}, \mathbf{p}_{a}\right)$. The length of the momentum four-vector is $m_{a} c$ so that $p_{a}^{0}=\sqrt{\left|\mathbf{p}_{a}\right|^{2}+m_{a}^{2} c^{2}}$.

An elastic collision between the particles of two constituent $a$ and $b$ whose momentum four-vectors before collision are denoted by $p_{a}^{\alpha}$ and $p_{b}^{\alpha}$ reads

$$
p_{a}^{\alpha}+p_{b}^{\alpha}=p_{a}^{\alpha}+p_{b}^{\alpha}
$$

where the primes denote the momentum four-vectors after collision.

A state of the relativistic mixture of $r$ constituents is characterized by the set of one-particle distribution functions

$$
f\left(\mathbf{x}, \mathbf{p}_{a}, t\right) \equiv f_{a}, \quad a=1,2, \ldots, r
$$

such that $f\left(\mathbf{x}, \mathbf{p}_{a}, t\right) d^{3} x d^{3} p_{a}$ gives at time $t$, the number of particles of constituent $a$ in the volume element $d^{3} x$ about $\mathbf{x}$ and with momenta in the range $d^{3} p_{a}$ about $\mathbf{p}_{a}$.

The one-particle distribution function of constituent a satisfies a Boltzmann equation, which in the absence of external fields reads (see e.g. $[16,17]$ )

$$
p_{a}^{\alpha} \partial_{\alpha} f_{a}=\sum_{b=1}^{r} \int\left(f_{a}^{\prime} f_{b}^{\prime}-f_{a} f_{b}\right) F_{b a} \sigma_{a b} d \Omega \frac{d^{3} p_{b}}{p_{b 0}},
$$

where $\sigma_{a b}$ and $d \Omega$ denote the invariant differential elastic cross-section and the element of solid angle that characterizes a binary collision between the particles of constituent $a$ with those of constituent $b$, respectively. Moreover, $F_{b a}$ is the invariant flux defined by

$$
F_{b a}=p_{a}^{0} p_{b}^{0} \sqrt{\left(\frac{\mathbf{p}_{a}}{p_{a}^{0}}-\frac{\mathbf{p}_{b}}{p_{b}^{0}}\right)^{2}-\left(\frac{\mathbf{p}_{a}}{p_{a}^{0}} \times \frac{\mathbf{p}_{b}}{p_{b}^{0}}\right)^{2}}=\sqrt{\left(p_{a}^{\alpha} p_{b \alpha}\right)^{2}-m_{a}^{2} m_{b}^{2} c^{4}},
$$

and the following abbreviations were introduced

$$
f_{a}^{\prime} \equiv f\left(\mathbf{x}, \mathbf{p}_{a}^{\prime}, t\right), \quad f_{b}^{\prime} \equiv f\left(\mathbf{x}, \mathbf{p}_{b}^{\prime}, t\right), \quad f_{a} \equiv f\left(\mathbf{x}, \mathbf{p}_{a}, t\right), \quad f_{b} \equiv f\left(\mathbf{x}, \mathbf{p}_{b}, t\right) .
$$

The general equation of transfer for the constituent $a$ of the mixture is obtained through the multiplication of the Boltzmann equation (2.3) by an arbitrary function of the momentum four-vector $\psi_{a} \equiv \psi\left(p_{a}^{\alpha}\right)$ and integration of the resulting equation over all values of $d^{3} p_{a} / p_{a 0}$, yielding:

$$
\begin{aligned}
\partial_{\alpha} \int \psi_{a} p_{a}^{\alpha} f_{a} \frac{d^{3} p_{a}}{p_{a 0}} & =\sum_{b=1}^{r} \int \psi_{a}\left(f_{a}^{\prime} f_{b}^{\prime}-f_{a} f_{b}\right) F_{b a} \sigma_{a b} d \Omega \frac{d^{3} p_{b}}{p_{b 0}} \frac{d^{3} p_{a}}{p_{a 0}} \\
& =\sum_{b=1}^{r} \int\left(\psi_{a}^{\prime}-\psi_{a}\right) f_{a} f_{b} F_{b a} \sigma_{a b} d \Omega \frac{d^{3} p_{b}}{p_{b 0}} \frac{d^{3} p_{a}}{p_{a 0}}
\end{aligned}
$$

where the last equality on the right-hand side of the above equation follows by using the symmetry properties of the collision term.

If we sum (2.6) over all constituents we get a general equation of transfer for the mixture that reads

$$
\partial_{\alpha} \sum_{a=1}^{r} \int \psi_{a} p_{a}^{\alpha} f_{a} \frac{d^{3} p_{a}}{p_{a 0}}=\frac{1}{2} \sum_{a, b=1}^{r} \int\left(\psi_{a}^{\prime}+\psi_{b}^{\prime}-\psi_{a}-\psi_{b}\right) f_{a} f_{b} F_{b a} \sigma_{a b} d \Omega \frac{d^{3} p_{b}}{p_{b 0}} \frac{d^{3} p_{a}}{p_{a 0}} .
$$

The right-hand side of (2.7) was also obtained by using the symmetry properties of the collision term and by interchanging the dummy indexes $a$ and $b$ in the sums.

The moments of the distribution function we are interested are the partial particle four-flow $N_{a}^{\alpha}$, the partial energymomentum tensor $T_{a}^{\alpha \beta}$ and the partial third-order moment tensor $T_{a}^{\alpha \beta \gamma}$ which are defined in terms of the distribution function through:

$$
N_{a}^{\alpha}=c \int p_{a}^{\alpha} f_{a} \frac{d^{3} p_{a}}{p_{a 0}}, \quad T_{a}^{\alpha \beta}=c \int p_{a}^{\alpha} p_{a}^{\beta} f_{a} \frac{d^{3} p_{a}}{p_{a 0}}, \quad T_{a}^{\alpha \beta \gamma}=c \int p_{a}^{\alpha} p_{a}^{\beta} p_{a}^{\gamma} f_{a} \frac{d^{3} p_{a}}{p_{a 0}} .
$$


The particle four-flow of the mixture $N^{\alpha}$, the energy-momentum tensor $T^{\alpha \beta}$ and the third-order moment $T^{\alpha \beta \gamma}$ of the mixture are obtained by summing the partial quantities over all constituents, i.e.

$$
N^{\alpha}=\sum_{a=1}^{r} N_{a}^{\alpha}, \quad T^{\alpha \beta}=\sum_{a=1}^{r} T_{a}^{\alpha \beta}, \quad T^{\alpha \beta \gamma}=\sum_{a=1}^{r} T_{a}^{\alpha \beta \gamma} .
$$

The balance equations for the partial particle four-flow, for the partial energy-momentum tensor and for the partial third-order moment tensor are obtained by choosing $\psi_{a}=c, \psi_{a}=c p_{a}^{\beta}$ and $\psi_{a}=c p_{a}^{\beta} p_{a}^{\gamma}$ in (2.6), yielding

$$
\partial_{\alpha} N_{a}^{\alpha}=0, \quad \partial_{\alpha} T_{a}^{\alpha \beta}=\mathrm{P}_{a}^{\beta}, \quad \partial_{\alpha} T_{a}^{\alpha \beta \gamma}=\mathrm{P}_{a}^{\beta \gamma},
$$

respectively. Above, the production terms $\mathrm{P}_{a}^{\beta}$ and $\mathrm{P}_{a}^{\beta \gamma}$ are given by

$$
\begin{array}{r}
\mathrm{P}_{a}^{\beta}=\sum_{b=1}^{r} c \int\left(p_{a}^{\prime \beta}-p_{a}^{\beta}\right) f_{a} f_{b} F_{b a} \sigma_{a b} d \Omega \frac{d^{3} p_{b}}{p_{b 0}} \frac{d^{3} p_{a}}{p_{a 0}} \\
\mathrm{P}_{a}^{\beta \gamma}=\sum_{b=1}^{r} c \int\left(p_{a}^{\prime \beta} p_{a}^{\prime \gamma}-p_{a}^{\beta} p_{a}^{\gamma}\right) f_{a} f_{b} F_{b a} \sigma_{a b} d \Omega \frac{d^{3} p_{b}}{p_{b 0}} \frac{d^{3} p_{a}}{p_{a 0}} .
\end{array}
$$

By summing (2.10) over all constituents of the mixture we get the balance equations for the particle four-flow, energy-momentum tensor and third-order moment tensor of the mixture that read

$$
\partial_{\alpha} N^{\alpha}=0, \quad \partial_{\alpha} T^{\alpha \beta}=0, \quad \partial_{\alpha} T^{\alpha \beta \gamma}=\mathrm{P}^{\beta \gamma}=\sum_{a=1}^{r} \mathrm{P}_{a}^{\beta \gamma}
$$

These are the balance equations used in the relativistic theory of extended thermodynamics of a single fluid (see [21]).

\section{ECKART DECOMPOSITION}

For the decomposition of Eckart we introduce the four-velocity $U^{\alpha}$ such that $U^{\alpha} U_{\alpha}=c^{2}$ and the projector

$$
\Delta^{\alpha \beta}=\eta^{\alpha \beta}-\frac{1}{c^{2}} U^{\alpha} U^{\beta}, \quad \text { with } \quad \Delta^{\alpha \beta} U_{\alpha}=0
$$

Hence, we write the partial particle four-flow as

$$
N_{a}^{\alpha}=\mathrm{n}_{a} U^{\alpha}+\mathrm{J}_{a}^{\alpha}, \text { such that } \mathrm{J}_{a}^{\alpha} U_{\alpha}=0 .
$$

Here $\mathrm{n}_{a}$ is the particle number density and $\mathrm{J}_{a}^{\alpha}$ the diffusion flux of the constituent $a$ in the mixture. They are defined in terms of the following projections of the partial particle four-flow

$$
\mathrm{n}_{a}=\frac{1}{c^{2}} N_{a}^{\alpha} U_{\alpha}, \quad \mathrm{J}_{a}^{\alpha}=\Delta_{\beta}^{\alpha} N_{a}^{\beta} .
$$

From the definitions (2.8a) and (3.2) we can represent the diffusion flux as

$$
\mathrm{J}_{a}^{\alpha}=\Delta_{\beta}^{\alpha} c \int p_{a}^{\beta} f_{a} \frac{d^{3} p_{a}}{p_{a 0}} .
$$

Further by summing (3.2) over all constituents it follows

$$
\mathrm{n}=\sum_{a=1}^{r} \mathrm{n}_{a}, \quad \sum_{a=1}^{r} \mathrm{~J}_{a}^{\alpha}=0
$$

which gives the particle number density of the mixture as the sum of the partial particle number densities and shows - due to the constraint (3.5b) - that there exist only $(r-1)$ partial diffusion fluxes that are linearly independent for a mixture of $r$ constituents.

The decomposition of the partial energy-momentum tensor in the Eckart description is written as (see e.g. [22-24])

$$
T_{a}^{\alpha \beta}=\mathrm{p}_{a}^{\langle\alpha \beta\rangle}-\left(\mathrm{p}_{a}+\varpi_{a}\right) \Delta^{\alpha \beta}+\frac{1}{c^{2}} U^{\alpha}\left(\mathrm{q}_{a}^{\beta}+\mathrm{h}_{a} \mathrm{~J}_{a}^{\beta}\right)+\frac{1}{c^{2}} U^{\beta}\left(\mathrm{q}_{a}^{\alpha}+\mathrm{h}_{a} \mathrm{~J}_{a}^{\alpha}\right)+\frac{\mathrm{n}_{a} \mathrm{e}_{a}}{c^{2}} U^{\alpha} U^{\beta},
$$


where we have introduced the partial quantities: $\mathrm{p}_{a}$ hydrostatic pressure, $\varpi_{a}$ non-equilibrium pressure, $\mathrm{e}_{a}$ energy per particle, $\mathrm{q}_{a}^{\alpha}$ heat flux, $\mathrm{h}_{a}=\mathrm{e}_{a}+\mathrm{p}_{a} / \mathrm{n}_{a}$ enthalpy per particle and $\mathrm{p}_{a}^{\langle\alpha \beta\rangle}$ pressure deviator tensor, i.e., the traceless part of the pressure tensor. These fields are given in terms of the projections of the partial energy-momentum tensor by:

$$
\begin{gathered}
\mathrm{e}_{a}=\frac{1}{\mathrm{n}_{a} c^{2}} U_{\alpha} T_{a}^{\alpha \beta} U_{\beta}, \quad \mathrm{p}_{a}+\varpi_{a}=-\frac{1}{3} \Delta_{\alpha \beta} T_{a}^{\alpha \beta}, \\
\mathrm{q}_{a}^{\alpha}+\mathrm{h}_{a} J_{a}^{\alpha}=\Delta_{\gamma}^{\alpha} T_{a}^{\gamma \beta} U_{\beta}, \quad \mathrm{p}_{a}^{\langle\alpha \beta\rangle}=\left(\Delta_{\gamma}^{\alpha} \Delta_{\delta}^{\beta}-\frac{1}{3} \Delta^{\alpha \beta} \Delta_{\gamma \delta}\right) T_{a}^{\gamma \delta} .
\end{gathered}
$$

Note that the symmetric and traceless part of a second order tensor $\Upsilon^{\alpha \beta}$ is defined in terms of the projector $\Delta^{\alpha \beta}$ through the relationship

$$
\Upsilon^{\langle\alpha \beta\rangle}=\left[\frac{\left(\Delta_{\gamma}^{\alpha} \Delta_{\delta}^{\beta}+\Delta_{\delta}^{\alpha} \Delta_{\gamma}^{\beta}\right)}{2}-\frac{\Delta^{\alpha \beta} \Delta_{\gamma \delta}}{3}\right] \Upsilon^{\gamma \delta} .
$$

If we sum the partial energy-momentum tensors over all constituents of the mixture and compare the resulting equation with the energy-momentum tensor of the mixture

$$
T^{\alpha \beta}=\mathrm{p}^{\langle\alpha \beta\rangle}-(\mathrm{p}+\varpi) \Delta^{\alpha \beta}+\frac{1}{c^{2}}\left(U^{\alpha} \mathrm{q}^{\beta}+U^{\beta} \mathrm{q}^{\alpha}\right)+\frac{\mathrm{ne}}{c^{2}} U^{\alpha} U^{\beta}
$$

we can identify the quantities of the mixture

$$
\begin{array}{r}
\mathrm{p}^{\langle\alpha \beta\rangle}=\sum_{a=1}^{r} \mathrm{p}_{a}^{\langle\alpha \beta\rangle}, \quad \mathrm{p}=\sum_{a=1}^{r} \mathrm{p}_{a}, \quad \varpi=\sum_{a=1}^{r} \varpi_{a}, \quad \mathrm{ne}=\sum_{a=1}^{r} \mathrm{n}_{a} \mathrm{e}_{a}, \\
\mathrm{nh}=\sum_{a=1}^{r} \mathrm{n}_{a} \mathrm{~h}_{a}, \quad \mathrm{q}^{\alpha}=\sum_{a=1}^{r}\left(\mathrm{q}_{a}^{\alpha}+\mathrm{h}_{a} \mathrm{~J}_{a}^{\alpha}\right) .
\end{array}
$$

Note that according to $(3.12 \mathrm{~b})$ the heat flux of the mixture is a sum of the partial heat fluxes and a term which represents the transport of heat due to diffusion.

\section{GRAD'S DISTRIBUTION FUNCTION}

By supposing that all constituents are at the same temperature $T$ - which represents the mixture's temperature we may describe a relativistic gas mixture by $13 r+1$ basic scalar fields:

$$
\begin{cases}\mathrm{n}_{a}, & r \text { scalar fields; } \\ U^{\alpha}, & 3 \text { scalar fields; } \\ \mathrm{J}_{a}^{\alpha}, & 3(r-1) \text { scalar fields; } \\ T, & 1 \text { scalar field; } \\ \varpi_{\alpha}, & r \text { scalar fields; } \\ \mathrm{q}_{a}^{\alpha}, & 3 r \text { scalar fields; } \\ \mathrm{p}_{a}^{\langle\alpha \beta\rangle}, & 5 r \text { scalar fields. }\end{cases}
$$

Unlike the non-relativistic theory of gases, the temperature in the relativistic theory cannot be defined in terms of the distribution function. It appears as the reciprocal of the integrating factor of the Pfaffian form $d e-\mathrm{p} d \mathrm{n} / \mathrm{n}^{2}$, which identifies the potential of the Gibbs equation in equilibrium with the entropy per particle $\mathbf{s}$ such that $d \mathbf{s}=$ $\frac{1}{T}\left(d \mathrm{e}-\frac{\mathrm{p}}{\mathrm{n}^{2}} d \mathrm{n}\right)$. Furthermore, in this work we are interested in process close to equilibrium where $\mathrm{J}_{a}^{\alpha}, \varpi_{a}, \mathrm{q}_{a}^{\alpha}$ and $\mathrm{p}_{a}^{\langle\alpha \beta\rangle}$ are considered as small quantities.

In terms of the $13 r+1$ basic fields the distribution function of constituent a can be represented as the following polynomial function of the momentum four-vector:

$$
f_{a}=f_{a}^{(0)}\left(1+A_{a}^{\alpha} p_{a \alpha}+A_{a}^{\alpha \beta} p_{a \alpha} p_{a \beta}\right)
$$

where $A_{a}^{\alpha}$ and $A_{a}^{\alpha \beta}$ are tensorial coefficients to be determined from the definitions of the partial particle four-flow (2.8a) and partial energy-momentum tensor (2.8b). Furthermore, $f_{a}^{(0)}$ is the Maxwell-Jüttner distribution function

$$
f_{a}^{(0)}=\frac{\mathrm{n}_{a}}{4 \pi k T m_{a}^{2} c K_{2}\left(\zeta_{a}\right)} \exp \left(-\frac{p_{a}^{\alpha} U_{\alpha}}{k T}\right) .
$$


Above $k$ is the Boltzmann constant, $K_{2}\left(\zeta_{a}\right)$ denotes the modified Bessel function of second kind defined through the integral

$$
K_{n}(\zeta)=\left(\frac{\zeta}{2}\right)^{n} \frac{\Gamma(1 / 2)}{\Gamma(n+1 / 2)} \int_{1}^{\infty} e^{-\zeta y}\left(y^{2}-1\right)^{n-1 / 2} d y
$$

and $\zeta_{a}=m_{a} c^{2} / k T$ is a parameter which represents the ratio of the rest energy $m_{a} c^{2}$ of a relativistic $a-$ particle and the thermal energy of the mixture $k T$. If $\zeta_{a} \gg 1$ the constituent behaves as a non-relativistic gas, while when $\zeta_{a} \ll 1$ as an ultra-relativistic gas.

In order to determine the tensorial coefficients we decompose the momentum four-vector $p_{a}^{\alpha}$ and the tensorial coefficients $A_{a}^{\alpha}$ and $A_{a}^{\alpha \beta}$ as

$$
\begin{array}{r}
p_{a}^{\alpha}=\Delta_{\beta}^{\alpha} p_{a}^{\beta}+\frac{1}{c^{2}} U^{\alpha}\left(p_{a}^{\beta} U_{\beta}\right), \quad A_{a}^{\alpha}=\lambda_{1} U^{\alpha}+\Lambda_{1}^{\beta} \Delta_{\beta}^{\alpha}, \\
A_{a}^{\alpha \beta}=\lambda_{2} U^{\alpha} U^{\beta}+\lambda_{3} \eta^{\alpha \beta}+\frac{1}{2} \Lambda_{2}^{\gamma}\left(\Delta_{\gamma}^{\alpha} U^{\beta}+\Delta_{\gamma}^{\beta} U^{\alpha}\right)+\Lambda^{\langle\gamma \delta\rangle} \Delta_{\gamma}^{\alpha} \Delta_{\delta}^{\beta} .
\end{array}
$$

Here we have introduced new coefficients: the scalars $\lambda_{1}, \lambda_{2}$ and $\lambda_{3}$, the four-vectors $\Lambda_{1}^{\alpha}$ and $\Lambda_{2}^{\alpha}$ and the symmetric and traceless second-order tensor $\Lambda^{\langle\gamma \delta\rangle}$.

Hence, the distribution function (4.2) can be written as

$$
\begin{array}{r}
f_{a}=f_{a}^{(0)}\left(1+\lambda_{1} p_{a}^{\alpha} U_{\alpha}+\lambda_{2} p_{a}^{\alpha} p_{a}^{\beta} U_{\alpha} U_{\beta}+\lambda_{3} m_{a}^{2} c^{2}+\Lambda_{1}^{\beta} p_{a}^{\alpha} \Delta_{\alpha \beta}\right. \\
\left.+\Lambda_{2}^{\gamma} p_{a}^{\alpha} p_{a}^{\beta} \Delta_{\gamma \alpha} U_{\beta}+\Lambda^{\langle\gamma \delta\rangle} p_{a}^{\alpha} p_{a}^{\beta} \Delta_{\alpha \gamma} \Delta_{\beta \delta}\right),
\end{array}
$$

thanks to (4.5) and (4.6).

Now the insertion of (4.7) into the definition of the partial particle four-flow (2.8a) and of the partial energymomentum tensor $(2.8 \mathrm{~b})$ and integration of the resulting equations leads to the determination of the lambda tensorial coefficients and it follows Grad's distribution function for the constituent $a$, namely,

$$
\begin{aligned}
f_{a} & =f_{a}^{(0)}\left\{1-\frac{\mathrm{J}_{a \alpha}}{\mathrm{p}_{a}} p_{a}^{\alpha}+\frac{\varpi_{a}}{\mathrm{p}_{a}} \frac{1-5 G_{a} \zeta_{a}-\zeta_{a}^{2}+G_{a}^{2} \zeta_{a}^{2}}{20 G_{a}+3 \zeta_{a}-13 G_{a}^{2} \zeta_{a}-2 G_{a} \zeta_{a}^{2}+2 G_{a}^{3} \zeta_{a}^{2}}\left[\frac{\zeta_{a}}{m_{a}^{2} c^{4}} U_{\alpha} U_{\beta} p_{a}^{\alpha} p_{a}^{\beta}\right.\right. \\
& \left.+\frac{3 \zeta_{a}}{m_{a} c^{2}} \frac{6 G_{a}+\zeta_{a}-G_{a}^{2} \zeta_{a}}{1-5 G_{a} \zeta_{a}-\zeta_{a}^{2}+G_{a}^{2} \zeta_{a}^{2}} U_{\alpha} p_{a}^{\alpha}+\frac{15 G_{a}+2 \zeta_{a}-6 G_{a}^{2} \zeta_{a}+5 G_{a} \zeta_{a}^{2}+\zeta_{a}^{3}-G_{a}^{2} \zeta_{a}^{3}}{1-5 G_{a} \zeta_{a}-\zeta_{a}^{2}+G_{a}^{2} \zeta_{a}^{2}}\right] \\
& \left.+\frac{\mathrm{q}_{a \alpha}}{\mathrm{p}_{a}} \frac{\zeta_{a}}{\zeta_{a}+5 G_{a}-G_{a}^{2} \zeta_{a}}\left[\frac{G_{a}}{m_{a} c^{2}} p_{a}^{\alpha}-\frac{1}{m_{a}^{2} c^{4}} U_{\beta} p_{a}^{\alpha} p_{a}^{\beta}\right]+\frac{\mathrm{p}_{a\langle\alpha \beta\rangle}}{\mathrm{p}_{a}} \frac{\zeta_{a}}{2 G_{a}} \frac{1}{m_{a}^{2} c^{2}} p_{a}^{\alpha} p_{a}^{\beta}\right\} .
\end{aligned}
$$

Above we have introduced the abbreviation $G_{a}=K_{3}\left(\zeta_{a}\right) / K_{2}\left(\zeta_{a}\right)$.

\section{CONSTITUTIVE EQUATIONS}

In order to determine the constitutive equation for the third-order moment tensor of constituent $a$ we insert Grad distribution function (4.8) into its definition (2.8c) and integrate the resulting equation, yielding

$$
\begin{aligned}
& T_{a}^{\alpha \beta \gamma}=\left(\mathrm{n}_{a} C_{1 a}+C_{2 a} \varpi_{a}\right) U^{\alpha} U^{\beta} U^{\gamma}+\frac{c^{2}}{6}\left(\mathrm{n}_{a} m_{a}^{2}-\mathrm{n}_{a} C_{1 a}-C_{2 a} \varpi_{a}\right)\left(\eta^{\alpha \beta} U^{\gamma}+\eta^{\alpha \gamma} U^{\beta}\right. \\
& \left.+\eta^{\beta \gamma} U^{\alpha}\right)+C_{3 a}\left(\eta^{\alpha \beta} \mathbf{q}_{a}^{\gamma}+\eta^{\alpha \gamma} \mathbf{q}_{a}^{\beta}+\eta^{\beta \gamma} \mathbf{q}_{a}^{\alpha}\right)-\frac{6}{c^{2}} C_{3 a}\left(U^{\alpha} U^{\beta} \mathbf{q}_{a}^{\gamma}+U^{\alpha} U^{\gamma} \mathbf{q}_{a}^{\beta}+U^{\beta} U^{\gamma} \mathbf{q}_{a}^{\alpha}\right) \\
& +C_{4 a}\left(\mathbf{p}_{a}^{\langle\alpha \beta\rangle} U^{\gamma}+\mathbf{p}_{a}^{\langle\alpha \gamma\rangle} U^{\beta}+\mathbf{p}_{a}^{\langle\beta \gamma\rangle} U^{\alpha}\right)+C_{5 a}\left(\eta^{\alpha \beta} \mathbf{J}_{a}^{\gamma}+\eta^{\alpha \gamma} \mathbf{J}_{a}^{\beta}+\eta^{\beta \gamma} \mathbf{J}_{a}^{\alpha}\right) \\
& +\frac{C_{6 a}}{c^{2}}\left(U^{\alpha} U^{\beta} \mathrm{J}_{a}^{\gamma}+U^{\alpha} U^{\gamma} \mathrm{J}_{a}^{\beta}+U^{\beta} U^{\gamma} \mathrm{J}_{a}^{\alpha}\right) .
\end{aligned}
$$

The scalar coefficients $C_{1 a}$ through $C_{6 a}$ are given by

$$
\begin{array}{r}
C_{1 a}=\frac{m_{a}^{2}}{\zeta_{a}}\left(\zeta_{a}+6 G_{a}\right), \quad C_{3 a}=-\frac{m_{a}}{\zeta_{a}} \frac{\zeta_{a}+6 G_{a}-G_{a}^{2} \zeta_{a}}{\zeta_{a}+5 G_{a}-G_{a}^{2} \zeta_{a}}, \\
C_{2 a}=-\frac{6 m_{a}}{c^{2} \zeta_{a}} \frac{2 \zeta_{a}^{3}-5 \zeta_{a}+\left(19 \zeta_{a}^{2}-30\right) G_{a}-\left(2 \zeta_{a}^{3}-45 \zeta_{a}\right) G_{a}^{2}-9 \zeta_{a}^{2} G_{a}^{3}}{20 G_{a}+3 \zeta_{a}-13 G_{a}^{2} \zeta_{a}-2 \zeta_{a}^{2} G_{a}+2 \zeta_{a}^{2} G_{a}^{3}}, \\
C_{4 a}=\frac{m_{a}}{G_{a} \zeta_{a}}\left(\zeta_{a}+6 G_{a}\right), \quad C_{5 a}=-m_{a} k T G_{a}, \quad C_{6 a}=m_{a} k T\left(\zeta_{a}+6 G_{a}\right) .
\end{array}
$$


Furthermore, the pressure $\mathrm{p}_{a}$, the energy per particle $\mathrm{e}_{a}$ and the enthalpy per particle $\mathrm{h}_{a}$ of constituent $a$ read

$$
\mathrm{p}_{a}=\mathrm{n}_{a} k T, \quad \mathrm{e}_{a}=m_{a} c^{2}\left(G_{a}-\frac{1}{\zeta_{a}}\right), \quad \mathrm{h}_{a}=m_{a} c^{2} G_{a} .
$$

For the determination of the production terms (2.11) and (2.12) it is necessary to introduce the total momentum four-vector $P^{\alpha}$ and the relative momentum four-vector $Q^{\alpha}$ defined by (see e.g. [7])

$$
P^{\alpha} \equiv p_{a}^{\alpha}+p_{b}^{\alpha}, \quad P^{\prime \alpha} \equiv p_{a}^{\prime \alpha}+p_{b}^{\prime \alpha}, \quad Q^{\alpha}=p_{a}^{\alpha}-p_{b}^{\alpha}, \quad Q^{\prime \alpha}=p_{a}^{\prime \alpha}-p_{b}^{\prime \alpha} .
$$

From the above equations together with the momentum four-vector conservation law it follows that

$$
P^{\alpha}=P^{\prime \alpha}, \quad P^{\alpha} Q_{\alpha}=\left(m_{a}^{2}-m_{b}^{2}\right) c^{2}, \quad Q^{2}=P^{2}-2\left(m_{a}^{2}+m_{b}^{2}\right) c^{2},
$$

where $P^{2}=P^{\alpha} P_{\alpha}$ and $Q^{2}=-Q^{\alpha} Q_{\alpha}$ denote the magnitudes of the total and relative momentum four-vectors, respectively. The inverse transformations of (5.6) are

$$
p_{a}^{\alpha}=\frac{P^{\alpha}}{2}+\frac{Q^{\alpha}}{2}, \quad p_{b}^{\alpha}=\frac{P^{\alpha}}{2}-\frac{Q^{\alpha}}{2}, \quad p_{a}^{\prime \alpha}=\frac{P^{\alpha}}{2}+\frac{Q^{\prime \alpha}}{2}, \quad p_{b}^{\prime \alpha}=\frac{P^{\alpha}}{2}-\frac{Q^{\prime \alpha}}{2} .
$$

The relative momentum four-vector can be written as

$$
Q^{\alpha}=\frac{\left(m_{a}^{2}-m_{b}^{2}\right) c^{2}}{P^{2}} P^{\alpha}+\frac{\mathrm{k}^{\alpha}}{P} \sqrt{P^{4}-2 P^{2}\left(m_{a}^{2}+m_{b}^{2}\right) c^{2}+\left(m_{a}^{2}-m_{b}^{2}\right)^{2} c^{4}},
$$

where $\mathrm{k}^{\alpha}$ is a spacelike unit vector orthogonal to $P^{\alpha}$, i.e., $\mathrm{k}^{\alpha} P_{\alpha}=0$.

From now on we shall restrict ourselves to the case where the rest masses of the particles of the constituents are not too disparate so that $m_{b} \approx m_{a}(1+\epsilon),(\forall b=1, \ldots, r \neq a)$ with $\epsilon$ denoting a small quantity. In this case we have that $m_{a}^{2}-m_{b}^{2} \approx\left(m_{a}+m_{b}\right) \epsilon$ and the term $\left(m_{a}^{2}-m_{b}^{2}\right)^{2}$ in (5.9) can be neglected. In this case the relative momentum four-vector can be approximated by

$$
Q^{\alpha}=\frac{\left(m_{a}^{2}-m_{b}^{2}\right) c^{2}}{P^{2}} P^{\alpha}+Q \mathrm{k}^{\alpha}
$$

and the invariant flux (2.4) reduces to

$$
F_{b a}=\sqrt{\left(p_{a}^{\alpha} p_{b \alpha}\right)^{2}-m_{a}^{2} m_{b}^{2} c^{4}}=\frac{P Q}{2} .
$$

For the determination of the production terms (2.11) and (2.12) we follow [17, 20] and introduce the invariant differential elastic cross-section for Maxwellian particles which can be written as

$$
\sigma_{a b}=\frac{P}{2 c Q} \mathcal{F}(\Theta)
$$

where $\mathcal{F}(\Theta)$ is an arbitrary function of the scattering angle $\Theta$. By taking into account the expression (5.11) and (5.12) we obtain that

$$
F_{b a} \sigma_{a b}=\frac{P^{2}}{4 c} \mathcal{F}(\Theta)
$$

This methodology is similar to the one employed in the non-relativistic case and the simple expression (5.13), which does not depend on the relative momentum four-vector, will permit us to integrate the production terms without the use of Grad's distribution function.

The calculation of the production terms is schematized in the Appendix A. Here we list only the final results

$$
\begin{aligned}
& \mathrm{P}_{a}^{\beta}=-\frac{\pi}{2 c^{2}} \mathcal{B}_{1} \sum_{b=1}^{r}\left[\left(T_{a}^{\beta \alpha}-T_{a \gamma}^{\gamma} \eta^{\beta \alpha}\right) N_{b \alpha}-\left(T_{b}^{\beta \alpha}-T_{b \gamma}^{\gamma} \eta^{\beta \alpha}\right) N_{a \alpha}\right], \\
& \mathrm{P}_{a}^{\alpha \beta}=-\frac{\pi}{2 c^{2}} \mathcal{B}_{1} \sum_{b=1}^{r}\left\{T_{a}^{\alpha \beta \gamma} N_{b \gamma}-T_{b}^{\alpha \beta \gamma} N_{a \gamma}+T_{a}^{\alpha \beta} T_{b \gamma}^{\gamma}-T_{b}^{\alpha \beta} T_{a \gamma}^{\gamma}\right. \\
& \left.-\frac{\left(m_{a}^{2}-m_{b}^{2}\right) c^{2}}{2}\left(N_{a}^{\alpha} N_{b}^{\beta}+N_{a}^{\beta} N_{b}^{\alpha}\right)\right\}+\frac{\pi}{8 c^{2}} \mathcal{B}_{2} \sum_{b=1}^{r}\left\{\left(\frac{m_{a}^{2}}{m_{b}^{2}}-5\right) T_{a}^{\alpha \beta} T_{b \gamma}^{\gamma}\right. \\
& +\left(\frac{m_{b}^{2}}{m_{a}^{2}}-5\right) T_{b}^{\alpha \beta} T_{a \gamma}^{\gamma}+4\left(T_{a}^{\alpha \gamma} T_{b \gamma}^{\beta}+T_{b}^{\alpha \gamma} T_{a \gamma}^{\beta}\right)+\left(m_{a}^{2}+m_{b}^{2}\right) c^{2}\left(N_{a}^{\alpha} N_{b}^{\beta}+N_{a}^{\beta} N_{b}^{\alpha}\right) \\
& \left.-2\left(T_{a}^{\alpha \beta \gamma} N_{b \gamma}+T_{b}^{\alpha \beta \gamma} N_{a \gamma}\right)-2 \eta^{\alpha \beta}\left[T_{a}^{\gamma \delta} T_{b \gamma \delta}-\left(\frac{m_{b}}{2 m_{a}}+\frac{m_{a}}{2 m_{b}}\right)^{2} T_{a \gamma}^{\gamma} T_{b \delta}^{\delta}\right]\right\},
\end{aligned}
$$


where we have introduced the following abbreviation for the integral

$$
\mathcal{B}_{n}=\int_{0}^{\pi} \mathcal{F}(\Theta)\left(1-\cos ^{n} \Theta\right) \sin \Theta d \Theta
$$

We note that with this methodology we were able to write the production terms (5.14) and (5.15) in terms on the moments of the distribution function $N_{a}^{\alpha}, T_{a}^{\alpha \beta}$ and $T_{a}^{\alpha \beta \gamma}$, without the knowledge of the distribution function.

\section{LINEARIZED $13 r+1$ FIELD EQUATIONS}

Once the constitutive equations for $T_{a}^{\alpha \beta \gamma}, \mathrm{P}_{a}^{\alpha}$ and $\mathrm{P}_{a}^{\alpha \beta}$ are given in terms of the $13 r+1$ fields (4.1) through (5.1), (5.14) and (5.15), respectively, we can obtain from the balance equations (2.10) the corresponding $13 r+1$ field equations. Here we are interested in the field equations that are linear in the non-equilibrium quantities $\mathrm{J}_{a}^{\alpha}, \varpi_{a}, \mathrm{q}_{a}^{\alpha}, \mathrm{p}_{a}^{\langle\alpha \beta\rangle}$ and their derivatives. Hence, the linearized field equations for the partial fields particle number density $\mathrm{n}_{a}$, fourvelocity $U^{\alpha}$ and temperature $T$ read

$$
\begin{aligned}
& \mathrm{Dn}_{a}+\mathrm{n}_{a} \nabla_{\alpha} U^{\alpha}+\nabla_{\alpha} \mathrm{J}_{a}^{\alpha}=0, \\
& \frac{\mathrm{nh}}{c^{2}} \mathrm{D} U^{\alpha}+\nabla_{\beta} \mathrm{p}^{\langle\alpha \beta\rangle}-\nabla^{\alpha}(\mathrm{p}+\varpi)+\frac{1}{c^{2}} \mathrm{Dq}^{\alpha}=0, \\
& \mathrm{n} c_{v} \mathrm{D} T+\mathrm{p} \nabla^{\alpha} U_{\alpha}+\nabla^{\alpha} \mathrm{q}_{\alpha}=0 .
\end{aligned}
$$

The balance equation of the particle number density (6.1) follows from (2.10a) by using (B2) of Appendix B. Note that we have introduced the operators $\mathrm{D}$ and $\nabla_{\alpha}$ defined through the relationships

$$
\mathrm{D}=U^{\alpha} \partial_{\alpha}, \quad \nabla_{\alpha}=\Delta_{\alpha}^{\beta} \partial_{\beta}, \quad \text { so that } \quad \partial_{\alpha}=\frac{1}{c^{2}} U_{\alpha} \mathrm{D}+\nabla_{\alpha} \quad \text { and } \quad U^{\alpha} \nabla_{\alpha}=0 .
$$

For the mixture the balance equations of the four-velocity (6.2) and of the temperature (6.3) follow from (2.10b), (B3) and (B5) of the Appendix B and by summing the resulting equation over all constituents. The former refers to the projection of this equation by $\Delta^{\alpha \beta}$, while the later is the projection with respect to $U^{\alpha}$. In (6.3) it was introduced the heat capacity per particle at constant volume of the mixture

$$
c_{v}=\left(\frac{\partial e}{\partial T}\right)_{v}=\sum_{a=1}^{r} k \frac{\mathrm{n}_{a}}{\mathrm{n}}\left[\zeta_{a}^{2}+5 G_{a} \zeta_{a}-G_{a}^{2} \zeta_{a}^{2}-1\right] .
$$

We get the balance equations for the partial diffusion fluxes $\mathrm{J}_{a}^{\alpha}$ from (2.10b), (B3) and (B5) of the Appendix B by taking the projection $\Delta_{\beta}^{\alpha}$ of the resulting equation and by subtracting the $r$ th equation from the $a$ th equation. This is necessary in order to obtain $3(r-1)$ independent scalar balance equations. Hence it follows

$$
\begin{aligned}
& \mathrm{D}\left(\frac{\mathrm{J}_{a}^{\alpha}}{\mathrm{n}_{a}}\right)-\mathrm{D}\left(\frac{\mathrm{J}_{r}^{\alpha}}{\mathrm{n}_{r}}\right)+\frac{c^{2}}{\mathrm{n}_{a} \mathrm{~h}_{a}}\left[\nabla_{\beta} \mathrm{p}_{a}^{\langle\alpha \beta\rangle}-\nabla^{\alpha}\left(\mathrm{p}_{a}+\varpi_{a}\right)+\frac{1}{c^{2}} \mathrm{Dq}_{a}^{\alpha}\right]-\frac{c^{2}}{\mathrm{n}_{r} \mathrm{~h}_{r}}\left[\nabla_{\beta} \mathrm{p}_{r}^{\langle\alpha \beta\rangle}+\frac{1}{c^{2}} \mathrm{Dq}_{r}^{\alpha}\right. \\
& \left.-\nabla^{\alpha}\left(\mathrm{p}_{r}+\varpi_{r}\right)\right]=-\frac{\pi}{2 \mathrm{n}_{a} \mathrm{~h}_{a}} \mathcal{B}_{1} \sum_{b=1}^{r}\left\{\left[\mathrm{~h}_{a}+\mathrm{h}_{b}-3 k T\right]\left(\mathrm{n}_{b} \mathrm{~J}_{a}^{\alpha}-\mathrm{n}_{a} \mathrm{~J}_{b}^{\alpha}\right)+\mathrm{n}_{b} \mathrm{q}_{a}^{\alpha}-\mathrm{n}_{a} \mathrm{q}_{b}^{\alpha}\right\} \\
& \quad-\frac{\pi}{2 \mathrm{n}_{r} \mathrm{~h}_{r}} \mathcal{B}_{1} \sum_{b=1}^{r}\left\{\left[\mathrm{~h}_{b}+\mathrm{h}_{r}-3 k T\right]\left(\mathrm{n}_{r} \mathrm{~J}_{b}^{\alpha}-\mathrm{n}_{b} \mathrm{~J}_{r}^{\alpha}\right)+\mathrm{n}_{r} \mathrm{q}_{b}^{\alpha}-\mathrm{n}_{b} \mathrm{q}_{r}^{\alpha}\right\} .
\end{aligned}
$$

The balance equations for the partial non-equilibrium pressures $\varpi_{a}$, heat fluxes $\mathrm{q}_{a}^{\alpha}$ and pressure deviator tensors $\mathrm{p}_{a}^{\langle\alpha \beta\rangle}$ are obtained from (2.10c), together with (B4) and (B6) of the Appendix B as follows: first the projection $U_{\alpha} U_{\beta}$ 
lead to the balance equations for the partial non-equilibrium pressures $\varpi_{a}$ :

$$
\begin{aligned}
& \frac{C_{2 a}}{2} \mathrm{D} \varpi_{a}+\frac{m_{a}^{2}+C_{1 a}}{2} \mathrm{Dn}_{a}-\frac{\zeta_{a}}{2 T} \mathrm{n}_{a} C_{1 a}^{\prime} \mathrm{D} T-5 \frac{C_{3 a}}{c^{2}} \nabla_{\gamma} \mathrm{q}_{a}^{\gamma}+\frac{\mathrm{n}_{a}}{6}\left(m_{a}^{2}+5 C_{1 a}\right) \nabla_{\gamma} U^{\gamma} \\
& +\frac{C_{5 a}+C_{6 a}}{c^{2}} \nabla_{\gamma} \mathrm{J}_{a}^{\gamma}=-\frac{\pi}{4 c^{4}} \mathcal{B}_{1} \sum_{b=1}^{r}\left[\mathrm{n}_{b}\left(c^{4} C_{2 a}+6 \mathrm{e}_{b}\right) \varpi_{a}-\mathrm{n}_{a}\left(c^{4} C_{2 b}+6 \mathrm{e}_{a}\right) \varpi_{b}\right] \\
& \quad-\frac{\pi}{8 c^{4}} \mathcal{B}_{2} \sum_{b=1}^{r}\left\{\mathrm { n } _ { b } \varpi _ { a } \left[c^{4} C_{2 a}-3\left(5-\frac{m_{b}^{2}}{m_{a}^{2}}\right) \mathrm{e}_{b}+6\left(\mathrm{e}_{b}-3 k T\right)\left(\frac{m_{a}}{2 m_{b}}+\frac{m_{b}}{2 m_{a}}\right)^{2}\right.\right. \\
& +6 k T]+\mathrm{n}_{a} \varpi_{b}\left[c^{4} C_{2 b}-3\left(5-\frac{m_{a}^{2}}{m_{b}^{2}}\right) \mathrm{e}_{a}+6\left(\mathrm{e}_{a}-3 k T\right)\left(\frac{m_{a}}{2 m_{b}}+\frac{m_{b}}{2 m_{a}}\right)^{2}\right. \\
& \quad+6 k T]+\frac{3 \mathrm{n}_{a} \mathrm{n}_{b}}{2}\left[\left(m_{a}^{2}-m_{b}^{2}\right)\left(\mathrm{e}_{a} \mathrm{e}_{b}-3(k T)^{2}\right)-3 k T\left(m_{a}^{2} \mathrm{e}_{a}-m_{b}^{2} \mathrm{e}_{b}\right)\right. \\
& \left.\left.\quad-k T\left(m_{a}^{2} \mathrm{e}_{b}-m_{b}^{2} \mathrm{e}_{a}\right)\right] \frac{m_{b}^{2}-m_{a}^{2}}{m_{a}^{2} m_{b}^{2}}\right\},
\end{aligned}
$$

where we have introduced $C_{1 a}^{\prime}=d C_{1 a}\left(\zeta_{a}\right) / d \zeta_{a}$. Next, the balance equations for the partial heat fluxes $\mathrm{q}_{a}^{\alpha}$ follow from the projection $\Delta_{\gamma}^{\alpha} U_{\beta}$ :

$$
\begin{aligned}
5 & C_{3 a} \mathrm{Dq}_{a}^{\alpha}-\frac{c^{4}}{6}\left[\left(m_{a}^{2}-C_{1 a}\right) \nabla^{\alpha} \mathrm{n}_{a}+\frac{\zeta_{a}}{T} \mathrm{n}_{a} C_{1 a}^{\prime} \nabla^{\alpha} T-C_{2 a} \nabla^{\alpha} \varpi_{a}\right]-c^{2} C_{4 a} \nabla_{\beta} \mathrm{p}_{a}^{\langle\alpha \beta\rangle} \\
& -\left(C_{5 a}+C_{6 a}\right) \mathrm{D} \mathrm{J}_{a}^{\alpha}-\frac{c^{2}}{6}\left(m_{a}^{2} \mathrm{n}_{a}+5 \mathrm{n}_{a} C_{1 a}\right) \mathrm{D} U^{\alpha}=-\frac{\pi}{2 c^{2}} \mathcal{B}_{1} \sum_{b=1}^{r}\left\{c ^ { 2 } \mathrm { n } _ { b } \mathrm { J } _ { a } ^ { \alpha } \left[C_{5 a}+C_{6 a}\right.\right. \\
& \left.-\frac{\left(m_{b}^{2}-C_{1 b}\right) c^{2}}{6}+\frac{\mathrm{h}_{a}}{c^{2}}\left(\mathrm{e}_{b}-3 k T\right)-\frac{\left(m_{a}^{2}-m_{b}^{2}\right) c^{2}}{2}\right]-c^{2} \mathrm{n}_{a} J_{b}^{\alpha}\left[C_{5 b}+C_{6 b}-\frac{\left(m_{a}^{2}-C_{1 a}\right) c^{2}}{6}\right. \\
& \left.+\frac{\mathrm{h}_{b}}{c^{2}}\left(\mathrm{e}_{a}-3 k T\right)+\frac{\left(m_{a}^{2}-m_{b}^{2}\right) c^{2}}{2}\right]+\mathrm{n}_{b}\left(5 c^{2} C_{3 a}-\mathrm{e}_{b}+3 k T\right) \mathrm{q}_{a}^{\alpha}-\mathrm{n}_{a}\left(5 c^{2} C_{3 b}-\mathrm{e}_{a}\right. \\
& \left.+3 k T) \mathrm{q}_{b}^{\alpha}\right\}-\frac{\pi}{8 c^{2}} \mathcal{B}_{2} \sum_{b=1}^{r}\left\{\mathrm{n}_{b}\left[10 C_{3 a} c^{2}+4\left(\mathrm{e}_{b}-k T\right)-\left(5-\frac{m_{a}^{2}}{m_{b}^{2}}\right)\left(\mathrm{e}_{b}-3 k T\right)\right] \mathrm{q}_{a}^{\alpha}\right. \\
& +\mathrm{n}_{a}\left[10 C_{3 b} c^{2}+4\left(\mathrm{e}_{a}-k T\right)-\left(5-\frac{m_{b}^{2}}{m_{a}^{2}}\right)\left(\mathrm{e}_{a}-3 k T\right)\right] \mathrm{q}_{b}^{\alpha}+2 c^{2} \mathrm{n}_{b}\left[C_{5 a}+C_{6 a}\right. \\
& \left.-\frac{\left(m_{b}^{2}-C_{1 b}\right) c^{2}}{6}+\left(5-\frac{m_{a}^{2}}{m_{b}^{2}}\right) \frac{\mathrm{e}_{b}-3 k T}{2} \frac{\mathrm{h}_{a}}{c^{2}}-2 \frac{\mathrm{h}_{a}}{c^{2}}\left(\mathrm{e}_{b}-k T\right)-\frac{\left(m_{a}^{2}+m_{b}^{2}\right) c^{2}}{2}\right] \mathrm{J}_{a}^{\alpha} \\
& +2 c^{2} \mathrm{n}_{a}\left[C_{5 b}+C_{6 b}-\frac{\left(m_{a}^{2}-C_{1 a}\right) c^{2}}{6}+\left(5-\frac{m_{b}^{2}}{m_{a}^{2}}\right) \frac{\mathrm{e}_{a}-3 k T}{2} \frac{\mathrm{h}_{b}}{c^{2}}-2 \frac{\mathrm{h}_{b}}{c^{2}}\left(\mathrm{e}_{a}-k T\right)\right. \\
& \left.\left.-\frac{\left(m_{a}^{2}+m_{b}^{2}\right) c^{2}}{2}\right] \mathrm{~J}_{b}^{\alpha}\right\} .
\end{aligned}
$$

Finally, the projection $\Delta_{\gamma}^{\alpha} \Delta_{\delta}^{\beta}-\Delta_{\gamma \delta} \Delta^{\alpha \beta} / 3$ imply the balance equations for the partial pressure deviator tensors $\mathrm{p}_{a}^{\langle\alpha \beta\rangle}$ :

$$
\begin{aligned}
& C_{4 a} \mathrm{D} \mathrm{p}_{a}^{\langle\alpha \beta\rangle}+2 C_{3 a} \nabla^{\langle\alpha} \mathrm{q}_{a}^{\beta\rangle}+2 C_{5 a} \nabla^{\langle\alpha} \mathrm{J}_{a}^{\beta\rangle}+\frac{c^{2}}{3} \mathrm{n}_{a}\left(m_{a}^{2}-C_{1 a}\right) \nabla^{\langle\alpha} U^{\beta\rangle} \\
& =-\frac{\pi}{2 c^{2}} \mathcal{B}_{1} \sum_{b=1}^{r}\left\{\mathrm{n}_{b}\left(c^{2} C_{4 a}+\mathrm{e}_{b}-3 k T\right) \mathrm{p}_{a}^{\langle\alpha \beta\rangle}-\mathrm{n}_{a}\left(c^{2} C_{4 b}+\mathrm{e}_{a}-3 k T\right) \mathrm{p}_{b}^{\langle\alpha \beta\rangle}\right\} \\
& \quad-\frac{\pi}{8 c^{2}} \mathcal{B}_{2} \sum_{b=1}^{r}\left\{\mathrm{n}_{b}\left[2 c^{2} C_{4 a}+\left(5-\frac{m_{a}^{2}}{m_{b}^{2}}\right)\left(\mathrm{e}_{b}-3 k T\right)+8 k T\right] \mathrm{p}_{a}^{\langle\alpha \beta\rangle}\right. \\
& \left.\quad+\mathrm{n}_{a}\left[2 c^{2} C_{4 b}+\left(5-\frac{m_{b}^{2}}{m_{a}^{2}}\right)\left(\mathrm{e}_{a}-3 k T\right)+8 k T\right] \mathrm{p}_{b}^{\langle\alpha \beta\rangle}\right\} .
\end{aligned}
$$

Hence, the system of equations (6.1), (6.2), (6.3), (6.6), (6.7), (6.8) and (6.9) compose 13r+1 linearized field equations for the fields (4.1). 


\section{SIX-FIELD THEORY FOR A BINARY MIXTURE}

In this section we shall restrict ourselves to a binary mixture characterized by the six scalar fields of particle number densities $\mathrm{n}_{1}, \mathrm{n}_{2}$, four-velocity $U^{\alpha}$ and temperature $T$, whose balance equations (6.1) - (6.3) are written as

$$
\begin{aligned}
& \mathrm{Dn}_{1}+\mathrm{n}_{1} \nabla_{\alpha} U^{\alpha}+\nabla_{\alpha} \mathrm{J}_{1}^{\alpha}=0, \quad \mathrm{Dn}_{2}+\mathrm{n}_{2} \nabla_{\alpha} U^{\alpha}+\nabla_{\alpha} \mathrm{J}_{2}^{\alpha}=0, \\
& \frac{\mathrm{nh}}{c^{2}} \mathrm{D} U^{\alpha}+\nabla_{\beta} \mathrm{p}^{\langle\alpha \beta\rangle}-\nabla^{\alpha}(\mathrm{p}+\varpi)+\frac{1}{c^{2}} \mathrm{Dq}^{\alpha}=0, \quad \mathrm{n} c_{v} \mathrm{D} T+\mathrm{p} \nabla^{\alpha} U_{\alpha}+\nabla^{\alpha} \mathrm{q}_{\alpha}=0 .
\end{aligned}
$$

In this case the pressure deviator tensors $\mathrm{p}_{1}^{\langle\alpha \beta\rangle}, \mathrm{p}_{2}^{\langle\alpha \beta\rangle}$, the non-equilibrium pressures $\varpi_{1}, \varpi_{2}$, the heat fluxes $\mathrm{q}_{1}^{\alpha}, \mathrm{q}_{2}^{\alpha}$ and the diffusion flux $J_{1}^{\alpha}=-J_{2}^{\alpha}$ are no longer variables, but constitutive quantities. To determine these constitutive quantities we shall rely on the remaining 21 scalar equations (6.6) - (6.9) and a method akin to the Maxwellian iteration procedure, which is often used in kinetic theory of gases. In this method the equilibrium values of the constitutive quantities - namely, $\mathrm{p}_{1}^{\langle\alpha \beta\rangle}=\mathrm{p}_{2}^{\langle\alpha \beta\rangle}=0, \varpi_{1}=\varpi_{2}=0, \mathrm{q}_{1}^{\alpha}=\mathrm{q}_{2}^{\alpha}=0$ and $\mathrm{J}_{1}^{\alpha}=-\mathrm{J}_{2}^{\alpha}=0$ - are inserted on the left-hand sides of the remaining 21 scalar equations and the first iterated values are obtained from the production terms, i.e., from the right-hand sides of these equations.

Following the methodology described in the Appendix $\mathrm{C}$ we get from:

(i) two equations that follow from (6.9)

$$
\mathrm{p}_{1}^{\langle\alpha \beta\rangle}=2 \mu_{1} \nabla^{\langle\alpha} U^{\beta\rangle}, \quad \mathrm{p}_{2}^{\langle\alpha \beta\rangle}=2 \mu_{2} \nabla^{\langle\alpha} U^{\beta\rangle} ;
$$

(ii) two equations that follow from (6.7)

$$
\varpi_{1}=-\eta_{1} \nabla^{\alpha} U_{\alpha}, \quad \varpi_{2}=-\eta_{2} \nabla^{\alpha} U_{\alpha} ;
$$

(iii) one equation and two equations that follow from (6.6) and (6.8), respectively,

$$
\mathrm{J}_{1}^{\alpha}=-\mathrm{J}_{2}^{\alpha}=\mathcal{D}_{12} \mathrm{~d}_{1}^{\alpha}+\mathcal{D}_{T} \nabla^{\alpha} \mathcal{T}, \quad \mathrm{q}_{1}^{\alpha}=\lambda_{1} \nabla^{\alpha} \mathcal{T}+\mathcal{D}_{1} \mathrm{~d}_{1}^{\alpha}, \quad \mathrm{q}_{2}^{\alpha}=\lambda_{2} \nabla^{\alpha} \mathcal{T}+\mathcal{D}_{2} \mathrm{~d}_{1}^{\alpha}
$$

Equations (7.3) and (7.4) represent the constitutive equations of a relativistic Newtonian fluid - also known as the Navier-Stokes law - and the associated transport coefficients $\mu_{1}, \mu_{2}$ and $\eta_{1}, \eta_{2}$ are identified as the coefficients of shear and bulk viscosities, respectively. In (7.5) we have introduced the generalized diffusion forces

$$
\mathrm{d}_{a}^{\alpha}=\frac{1}{k T}\left(\nabla^{\alpha} \mathrm{p}_{a}-\frac{\mathrm{n}_{a} \mathrm{~h}_{a}}{\mathrm{nh}} \nabla^{\alpha} \mathrm{p}\right), \quad a=1,2,
$$

that are restricted by the constraint $\sum_{a=1}^{2} \mathrm{~d}_{a}^{\alpha}=0$, so that $\mathrm{d}_{2}^{\alpha}=-\mathrm{d}_{1}^{\alpha}$, and the relativistic temperature gradient

$$
\nabla^{\alpha} \mathcal{T}=\left(\nabla^{\alpha} T-\frac{T}{\mathrm{nh}} \nabla^{\alpha} \mathrm{p}\right)
$$

which in the non-relativistic limiting case reduces to the temperature gradient $\nabla^{\alpha} T$. Hence, we may identify (7.5a) as the generalized Fick's law and $(7.5 \mathrm{~b}, \mathrm{c})$ as the generalized Fourier's law. The corresponding transport coefficients in these equations are recognized as the coefficients of diffusion $\mathcal{D}_{12}$, thermal-diffusion $\mathcal{D}_{T}$, thermal conductivity $\lambda_{1}, \lambda_{2}$ and diffusion-thermal $\mathcal{D}_{1}, \mathcal{D}_{2}$.

Now we are ready to obtain the constitutive equations necessary to convert the system of equations (7.1) and (7.2) into a system of field equations for the six fields of partial particle number densities $\mathrm{n}_{1}, \mathrm{n}_{2}$, four-velocity $U^{\alpha}$ and temperature $T$. Indeed, by using the definitions of the pressure deviator tensor, non-equilibrium pressure and heat flux of the mixture given by (3.11) and (3.12) we get

$$
\mathrm{p}^{\langle\alpha \beta\rangle}=2 \mu \nabla^{\langle\alpha} U^{\beta\rangle}, \quad \varpi=-\eta \nabla^{\alpha} U_{\alpha}, \quad \mathrm{q}^{\alpha}=\lambda^{\prime} \nabla^{\alpha} \mathcal{T}+\mathcal{D} \mathrm{d}_{1}^{\alpha} .
$$

Here the transport coefficients of shear viscosity $\mu$, bulk viscosity $\eta$ and thermal conductivity $\lambda^{\prime}$ of the mixture and the diffusion-thermal coefficient $\mathcal{D}$ read

$$
\begin{aligned}
& \mu=\mu_{1}+\mu_{2}, \quad \eta=\eta_{1}+\eta_{2}, \quad \lambda^{\prime}=\lambda_{1}+\lambda_{2}+\left(\mathrm{h}_{1}-\mathrm{h}_{2}\right) \mathcal{D}_{T}, \\
& \mathcal{D}=\mathcal{D}_{1}+\mathcal{D}_{2}+\left(\mathrm{h}_{1}-\mathrm{h}_{2}\right) \mathcal{D}_{12} .
\end{aligned}
$$

We call attention to the fact that the true thermal conductivity of the mixture is defined as the ratio of the heat flux and the temperature gradient when there is no diffusion, i.e., when $\mathrm{J}_{1}^{\alpha}=-\mathrm{J}_{2}^{\alpha}=0$. In this case the we get from (7.5) that

$$
\mathrm{d}_{1}^{\alpha}=-\frac{\mathcal{D}_{T}}{\mathcal{D}_{12}} \nabla^{\alpha} \mathcal{T}, \quad \text { hence } \quad \mathrm{q}^{\alpha}=\lambda \nabla^{\alpha} \mathcal{T}
$$


where the true thermal conductivity of the mixture is given by

$$
\lambda=\lambda_{1}+\lambda_{2}-\frac{\mathcal{D}_{T}}{\mathcal{D}_{12}}\left(\mathcal{D}_{1}+\mathcal{D}_{2}\right) .
$$

The constitutive relation for the diffusion flux (7.5a) together with the ones for the pressure deviator tensor, nonequilibrium pressure and heat flux (7.8) imply into the desired field equations for the six fields $\left(\mathrm{n}_{1}, \mathrm{n}_{2}, U^{\alpha}, T\right)$, when they are inserted into the system of equations (7.1) and (7.2).

The expressions for the transport coefficients, even in the order up to $\epsilon$, are too large to be given here. Bellow we present only their expressions in the non- and ultra-relativistic limiting cases.

In the non-relativistic limiting case the thermal energy of the gas $k T$ is smaller than the particle rest energy $m_{1} c^{2}$, so that $\zeta_{1} \gg 1$ and we obtain

$$
\begin{aligned}
\mathcal{D}_{12} & =\frac{k T}{\pi m_{1} \mathrm{n} \mathcal{B}_{1}}\left\{1-\frac{\left(\mathcal{B}_{1}+\mathcal{B}_{2}\right) \mathrm{n}+2 \mathrm{n}_{1} \mathcal{B}_{1}}{2 \mathrm{n}\left(2 \mathcal{B}_{1}+\mathcal{B}_{2}\right)} \epsilon-\frac{4 \mathcal{B}_{1}+\mathcal{B}_{2}}{2\left(2 \mathcal{B}_{1}+\mathcal{B}_{2}\right)}[1\right. \\
& \left.-\frac{\mathcal{B}_{1}^{2}\left(26 \mathrm{n}_{1}+6 \mathrm{n}_{2}\right)+\mathcal{B}_{2}^{2}\left(\mathrm{n}_{1}+3 \mathrm{n}_{2}\right)+\mathcal{B}_{1} \mathcal{B}_{2}\left(13 \mathrm{n}_{1}+11 \mathrm{n}_{2}\right)}{2 \mathrm{n}\left(2 \mathcal{B}_{1}+\mathcal{B}_{2}\right)\left(4 \mathcal{B}_{1}+\mathcal{B}_{2}\right)} \epsilon \frac{1}{\zeta_{1}}\right\}+\mathcal{O}\left(\epsilon^{2}, \frac{1}{\zeta_{1}^{2}}\right), \\
\mathcal{D}_{T} & =-\frac{5\left(\mathcal{B}_{1}+\mathcal{B}_{2}\right) k \mathrm{n}_{1} \mathrm{n}_{2}}{4 \pi m_{1} \mathrm{n}^{2} \mathcal{B}_{2}\left(2 \mathcal{B}_{1}+\mathcal{B}_{2}\right)}\left\{\frac{1}{\zeta_{1}}-\frac{5 \mathcal{B}_{1}^{2}+\mathcal{B}_{1} \mathcal{B}_{2}-\mathcal{B}_{2}^{2}}{\left(\mathcal{B}_{1}+\mathcal{B}_{2}\right)\left(2 \mathcal{B}_{1}+\mathcal{B}_{2}\right)} \frac{1}{\zeta_{1}^{2}}\right\} \epsilon+\mathcal{O}\left(\epsilon^{2}, \frac{1}{\zeta_{1}^{3}}\right), \\
\mathcal{D} & =-\frac{k T \mathrm{~h}_{1}^{0}}{\pi m_{1} \mathrm{n} \mathcal{B}_{1} \mathcal{B}_{2}}\left\{\mathcal{B}_{1}+\mathcal{B}_{2}-\frac{\mathcal{B}_{1}\left(6 \mathcal{B}_{1}+7 \mathcal{B}_{2}\right)}{4 \mathcal{B}_{1}+2 \mathcal{B}_{2}} \frac{1}{\zeta_{1}}\right\} \epsilon+\mathcal{O}\left(\epsilon^{2}, \frac{1}{\zeta_{1}^{2}}\right), \\
\mu & =\frac{2 k T}{3 \pi \mathcal{B}_{2}}\left\{1+\frac{1}{\zeta_{1}}\left(1-\frac{\mathrm{n}_{2}}{\mathrm{n}} \epsilon\right)-\frac{7}{3 \zeta_{1}^{2}}\left(1-2 \frac{\mathrm{n}_{2}}{\mathrm{n}} \epsilon\right)\right\}+\mathcal{O}\left(\epsilon^{2}, \frac{1}{\zeta_{1}^{3}}\right), \\
\eta & =\frac{5 k T}{6 \pi \mathcal{B}_{2}} \frac{1}{\zeta_{1}^{2}}\left\{1-2 \frac{\mathrm{n}_{2}}{\mathrm{n}} \epsilon-\frac{25}{2 \zeta_{1}}\left(1-3 \frac{\mathrm{n}_{2}}{\mathrm{n}} \epsilon\right)\right\}+\mathcal{O}\left(\epsilon^{2}, \frac{1}{\zeta_{1}^{4}}\right), \\
\lambda & =\lambda^{\prime}=\frac{5 k^{2} T}{2 \pi m_{1} \mathcal{B}_{2}}\left\{1-\frac{\mathrm{n}_{2}}{\mathrm{n}} \epsilon-\frac{39}{8 \zeta_{1}^{2}}\left(1-3 \frac{\mathrm{n}_{2}}{\mathrm{n}} \epsilon\right)\right\}+\mathcal{O}\left(\epsilon^{2}, \frac{1}{\zeta_{1}^{3}}\right),
\end{aligned}
$$

In the ultra-relativistic limiting case the thermal energy of the gas $k T$ is larger than the particle rest energy $m_{1} c^{2}$, so that $\zeta_{1} \ll 1$ and we have

$$
\begin{aligned}
\mathcal{D}_{12} & =\frac{6 c^{2}\left(2 \mathcal{B}_{1}+\mathcal{B}_{2}\right)}{\pi \mathrm{n} \mathcal{B}_{1}\left(26 \mathcal{B}_{1}+11 \mathcal{B}_{2}\right)}\left\{1-\frac{2\left(11 \mathcal{B}_{1}+5 \mathcal{B}_{2}\right)}{3\left(26 \mathcal{B}_{1}+11 \mathcal{B}_{2}\right)}[1\right. \\
& \left.\left.+\frac{4 \mathcal{B}_{1}^{2}\left(15 \mathrm{n}_{1}+7 \mathrm{n}_{2}\right)+\mathcal{B}_{1} \mathcal{B}_{2}\left(55 \mathrm{n}_{1}+29 \mathrm{n}_{2}\right)+\mathcal{B}_{2}^{2}\left(13 \mathrm{n}_{1}+7 \mathrm{n}_{2}\right)}{2 \mathrm{n}\left(11 \mathcal{B}_{1}+5 \mathcal{B}_{2}\right)\left(2 \mathcal{B}_{1}+\mathcal{B}_{2}\right)} \epsilon\right] \zeta_{1}^{2}\right\}+\mathcal{O}\left(\epsilon^{2}, \zeta_{1}^{3}\right) \\
\mathcal{D}_{T} & =-\frac{c^{2}\left(6 \mathcal{B}_{1}+11 \mathcal{B}_{2}\right) \mathrm{n}_{1} \mathrm{n}_{2}}{3 \pi \mathrm{n}^{2} T \mathcal{B}_{2}\left(26 \mathcal{B}_{1}+11 \mathcal{B}_{2}\right)} \epsilon \zeta_{1}^{2}+\mathcal{O}\left(\epsilon^{2}, \zeta_{1}^{3}\right) \\
\mathcal{D} & =-\frac{k T c^{2}}{\pi \mathrm{n} \mathcal{B}_{2}} \frac{26 \mathcal{B}_{1}^{2}+55 \mathcal{B}_{1} \mathcal{B}_{2}+26 \mathcal{B}_{2}^{2}}{78 \mathcal{B}_{1}+33 \mathcal{B}_{2}} \zeta_{1}^{2} \epsilon+\mathcal{O}\left(\epsilon^{2}, \zeta_{1}^{3}\right) \\
\mu & =\frac{4 k T}{5 \pi \mathcal{B}_{2}}\left\{1+\frac{\zeta_{1}^{4}}{80}\left[\frac{1}{4}+2 \frac{\mathrm{n}_{2}}{\mathrm{n}} \epsilon+\left(\gamma+\ln \frac{\zeta_{1}}{2}\right)\left(1+4 \frac{\mathrm{n}_{2}}{\mathrm{n}} \epsilon\right)\right]+\mathcal{O}\left(\epsilon^{2}, \zeta_{1}^{5}\right)\right. \\
\eta & =\frac{k T \mathrm{n}_{1}}{108 \pi \mathrm{n} \mathcal{B}_{2}} \zeta_{1}^{4}\left\{1+\frac{10 \mathrm{n}_{2}\left(22 \mathcal{B}_{1}+29 \mathcal{B}_{2}\right)}{\mathrm{n}\left(2 \mathcal{B}_{1}+\mathcal{B}_{2}\right)} \epsilon\right\}+\mathcal{O}\left(\epsilon^{2}, \zeta_{1}^{5}\right) \\
\lambda & =\lambda^{\prime}=\frac{4 c^{2} k}{3 \pi \mathcal{B}_{2}}\left\{1-\frac{\zeta_{1}^{2}}{3}\left(1+2 \frac{\mathrm{n}_{2}}{\mathrm{n}} \epsilon\right)\right\}+\mathcal{O}\left(\epsilon^{2}, \zeta_{1}^{3}\right)
\end{aligned}
$$

From the expressions of the transport coefficients $(7.13)-(7.23)$ we note that:

1. they are valid up to the $\epsilon$-order, which was the approximation used when the masses of the particles of the constituents are not too disparate, so that we have written $m_{2}=m_{1}(1+\epsilon)$ with $\epsilon$ being a small quantity;

2. in all expressions relativistic corrections are given in terms of the parameter $\zeta_{1}$;

3. the coefficients of thermal-diffusion $\mathcal{D}_{T}$ and diffusion-thermal $\mathcal{D}$ are of order $\epsilon$; 
4. the thermal-diffusion coefficient $\mathcal{D}_{T}$ given by (7.14) is of relativistic order, i.e., it vanishes in the non-relativistic limiting case. This is well known result in the literature that the thermal-diffusion coefficient vanishes for a mixture of Maxwellian particles (see e.g. [25, 26]);

5. the diffusion-thermal coefficient $\mathcal{D}$ is also of relativistic order, since its expression (7.15) depends on the rest energy $\mathrm{h}_{1}^{0}=m_{1} c^{2}$

6. within the $\epsilon$-order the coefficients $\lambda$ and $\lambda^{\prime}$, associated with the thermal conductivity of the mixture, coincide;

7. for a single component - i.e., when $\mathrm{x}_{2}=0$ - the transport coefficients of shear and bulk viscosities and thermal conductivity in the non-relativistic and ultra-relativistic limiting cases reduce to the ones given in [17, 20].

In the case of the particles have the same rest masses $m_{1}=m_{2}$ the coefficients of thermal-diffusion $\mathcal{D}_{T}$ and diffusion-thermal $\mathcal{D}$ vanish and the diffusion coefficient becomes the self-diffusion coefficient $\mathcal{D}_{11}$ whose expression is given by

$$
\begin{aligned}
& \mathcal{D}_{11}=\frac{-2 c^{2}\left(2 \mathcal{B}_{1}+\mathcal{B}_{2}\right)\left(10 G_{1}+\zeta_{1}-G_{1}^{2} \zeta_{1}\right)}{\Delta}, \quad \text { where } \\
& \begin{aligned}
\Delta & =\pi \mathrm{n} \mathcal{B}_{1}\left\{2 \mathcal{B}_{1}\left[G_{1}^{3} \zeta_{1}^{2}\left(G_{1} \zeta_{1}-8\right)+G_{1}\left(30+8 \zeta_{1}^{2}\right)+\zeta_{1}\left(3+\zeta_{1}^{2}\right)+2 G_{1}^{2} \zeta_{1}\left(1-\zeta_{1}^{2}\right)\right]\right. \\
& \left.+\mathcal{B}_{2}\left(30 G_{1}+3 \zeta_{1}-13 G_{1}^{2} \zeta_{1}\right)\right\} .
\end{aligned}
\end{aligned}
$$

When $m_{1}=m_{2}$ the character of the mixture is exclusively owing to the difference of the particle number densities of the components. Furthermore the coefficients of thermal conductivity, shear and bulk viscosities of the mixture reduce to

$$
\begin{array}{r}
\lambda=\frac{2 k c^{2} \zeta\left(\zeta+5 G-G^{2} \zeta\right)^{2}}{\pi \mathcal{B}_{2}\left(\zeta+10 G-\zeta G^{2}\right)}, \quad \mu=\frac{2 k T G^{2} \zeta}{\pi \mathcal{B}_{2}\left(2 G+\zeta+2 G^{2} \zeta\right)} \\
\eta=\frac{k T \zeta\left(20 G+3 \zeta-13 G^{2} \zeta-2 G \zeta^{2}+2 G^{3} \zeta^{2}\right)^{2}}{3 \pi \mathcal{B}_{2}\left(1-5 G \zeta-\zeta^{2}+G^{2} \zeta^{2}\right)^{2}\left(\zeta+5 G-\zeta G^{2}\right)}
\end{array}
$$

which are the same as those of a single gas $[17,20]$. The constitutive equations in the case where the masses of the particles are identical read

$$
\mathrm{J}_{1}^{\alpha}=-\mathrm{J}_{2}^{\alpha}=\mathcal{D}_{11} \mathrm{~d}_{1}^{\alpha}, \quad \mathrm{p}^{\langle\alpha \beta\rangle}=2 \mu \nabla^{\langle\alpha} U^{\beta\rangle}, \quad \varpi=-\eta \nabla^{\alpha} U_{\alpha}, \quad \mathrm{q}^{\alpha}=\lambda \nabla^{\alpha} \mathcal{T} .
$$

In the next section we shall investigate the solutions of the six-field theory concerning the propagation of forced and free waves in a relativistic binary mixture and for that end we shall need the values of the transport coefficients: diffusion $\mathcal{D}_{12}$ thermal-diffusion $\mathcal{D}_{T}$, thermal conductivity $\lambda^{\prime}$, diffusion-thermal $\mathcal{D}$, shear viscosity $\mu$ and bulk viscosity $\eta$.

In Figures $1-3$ are plotted the dimensionless coefficients

$$
\begin{array}{r}
\mathcal{D}_{12}^{*}=\mathcal{D}_{12} \frac{\mathcal{B}_{2} \mathrm{n}}{c^{2}}, \quad \mathcal{D}_{T}^{*}=\mathcal{D}_{T} \frac{\mathcal{B}_{2} T}{c^{2}}, \quad \lambda^{*}=\lambda^{\prime} \frac{\mathcal{B}_{2}}{k c^{2}}, \\
\mathcal{D}^{*}=\mathcal{D} \frac{\mathcal{B}_{2} \mathrm{n}}{k T c^{2}}, \quad \mu^{*}=\mu \frac{\mathcal{B}_{2}}{k T}, \quad \eta^{*}=\eta \frac{\mathcal{B}_{2}}{k T},
\end{array}
$$

as functions of the parameter $\zeta_{1}=m_{1} c^{2} / k T$. In these figures it was consider that the concentration of the constituent labeled with the index 1 was $60 \%$, i.e., $\mathrm{x}_{1}=\mathrm{n}_{1} / \mathrm{n}=0.6$ with $\mathrm{x}_{2}=1-\mathrm{x}_{1}$, and that the mass of the constituent 2 was $10 \%$ larger than that of the constituent 1 , so that $\epsilon=0.1$. Furthermore, the ratio of the integrals $\mathcal{B}_{2} / \mathcal{B}_{1}$ was taken equal to the ratio of the integrals that appear in the theory of non-relativistic Maxwellian particles, namely, $A_{2}(5) / A_{1}(5)$ (see, e.g. $[25,26]$ ). Hence, we have adopted the value $\mathcal{B}_{2} / \mathcal{B}_{1}=0.436 / 0.422$.

We infer from these figures for the dimensionless coefficients that:

1. the values of the diffusion $\mathcal{D}_{12}^{*}$, shear viscosity $\mu^{*}$ and thermal conductivity $\lambda^{*}$ in the non-relativistic limiting case are smaller than those in the ultra-relativistic one;

2. the coefficients of thermal-diffusion $\mathcal{D}_{T}^{*}$ and bulk viscosity $\eta^{*}$ vanish for very small and very large values of $\zeta_{1}$;

3. the diffusion-thermal coefficient $\mathcal{D}^{*}$ is very small in the ultra-relativistic limit and tends to a constant value in the non-relativistic limit. 

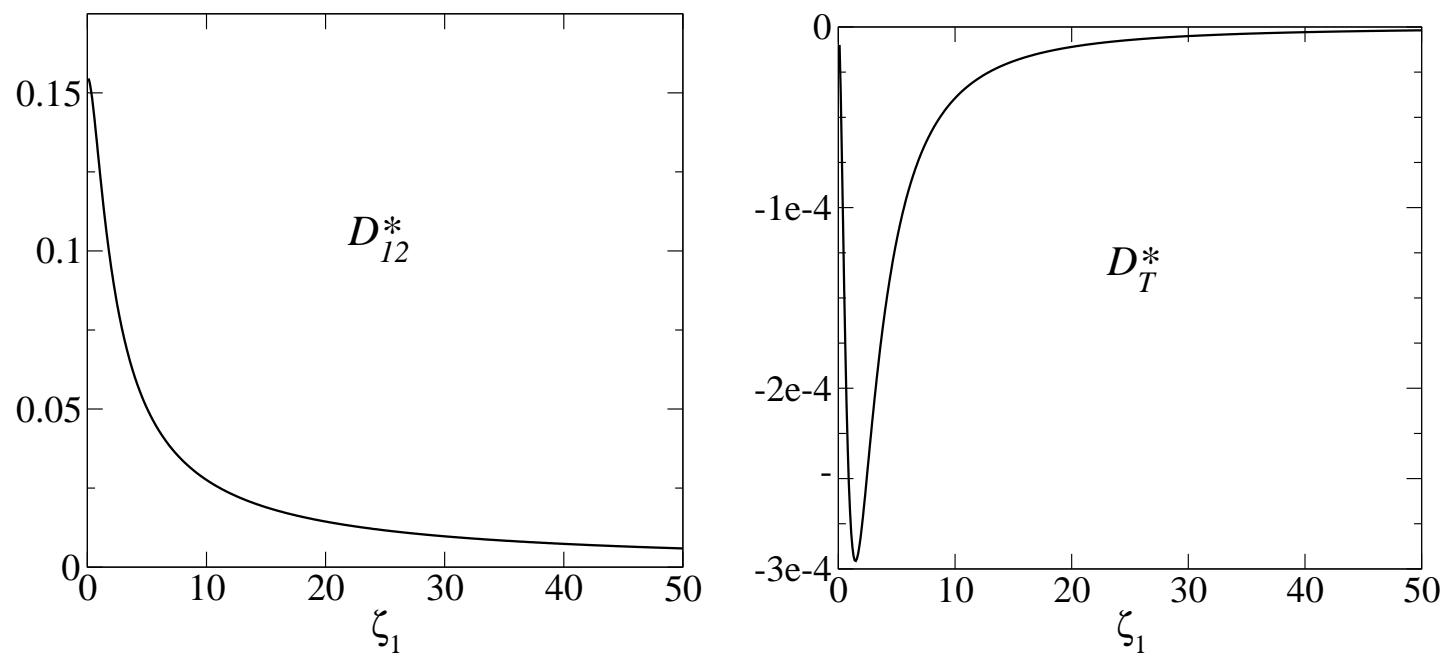

FIG. 1. Dimensionless coefficients as functions of $\zeta_{1}$ : diffusion $\mathcal{D}_{12}^{*}$ (fig. 1a) and thermal-diffusion $\mathcal{D}_{T}^{*}$ (fig. 1b).
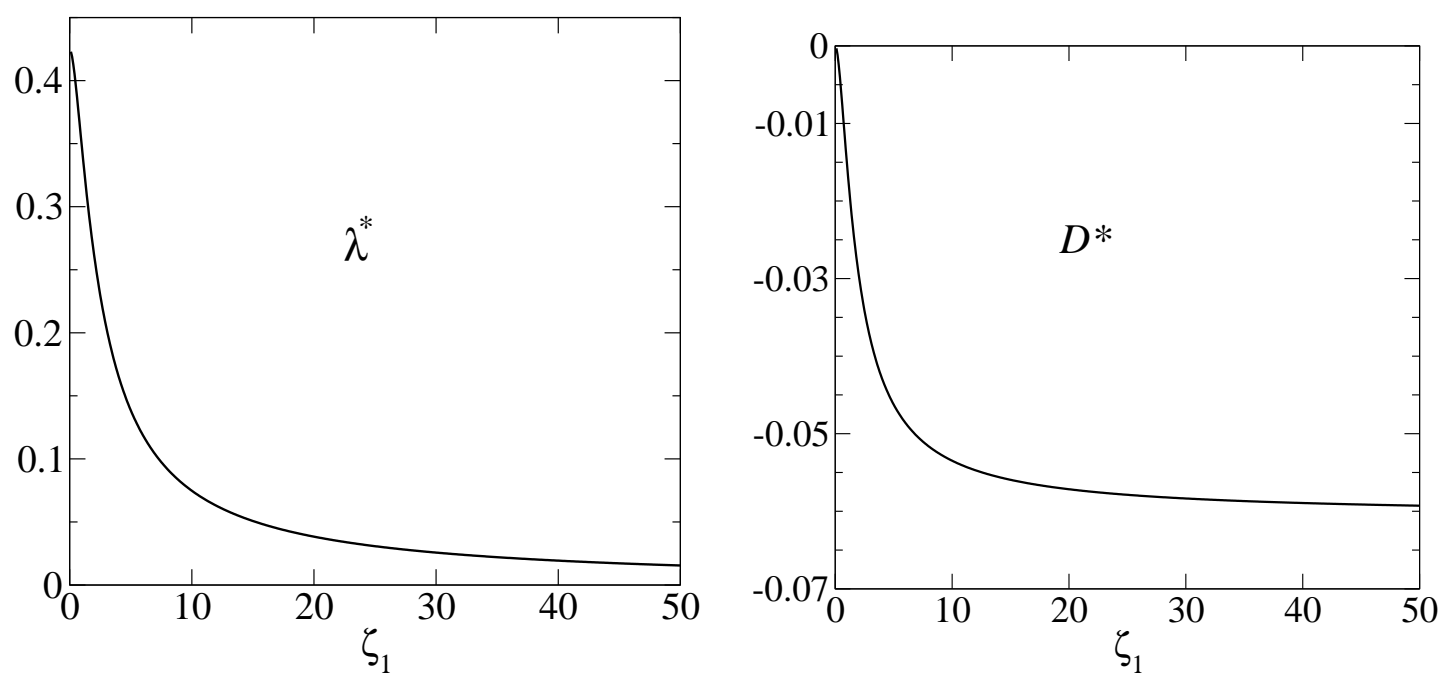

FIG. 2. Dimensionless coefficients as functions of $\zeta_{1}$ : thermal conductivity $\lambda^{*}$ (fig. 2a) and diffusion-thermal $\mathcal{D}^{*}$ (fig. $2 \mathrm{~b}$ ).

\section{FORCED AND FREE WAVES IN A RELATIVISTIC BINARY MIXTURE}

As was pointed out in the last section,, the insertion of the constitutive relations (7.5a) and (7.8) into the balance equations (7.1) and (7.2) leads to system of field equations for the determination of the six fields of partial particle number densities $\mathrm{n}_{a}(a=1,2)$, mixture four-velocity $U^{\alpha}$ and mixture temperature $T$. Let us then look for solutions of form

$$
\begin{array}{r}
\mathrm{n}_{a}=\times_{a} \mathrm{n}_{0}+\overline{\mathrm{n}}_{a} \exp (\imath q x-\imath \omega t), \quad T=T_{0}+\bar{T} \exp (\imath q x-\imath \omega t), \\
U^{\alpha}=\left(\frac{c}{\sqrt{1-[(\bar{v} / c) \exp (\imath q x-\imath \omega t)]^{2}}}, \frac{\bar{v} \exp (\imath q x-\imath \omega t)}{\sqrt{1-[(\bar{v} / c) \exp (\imath q x-\imath \omega t)]^{2}}}, 0,0\right),
\end{array}
$$



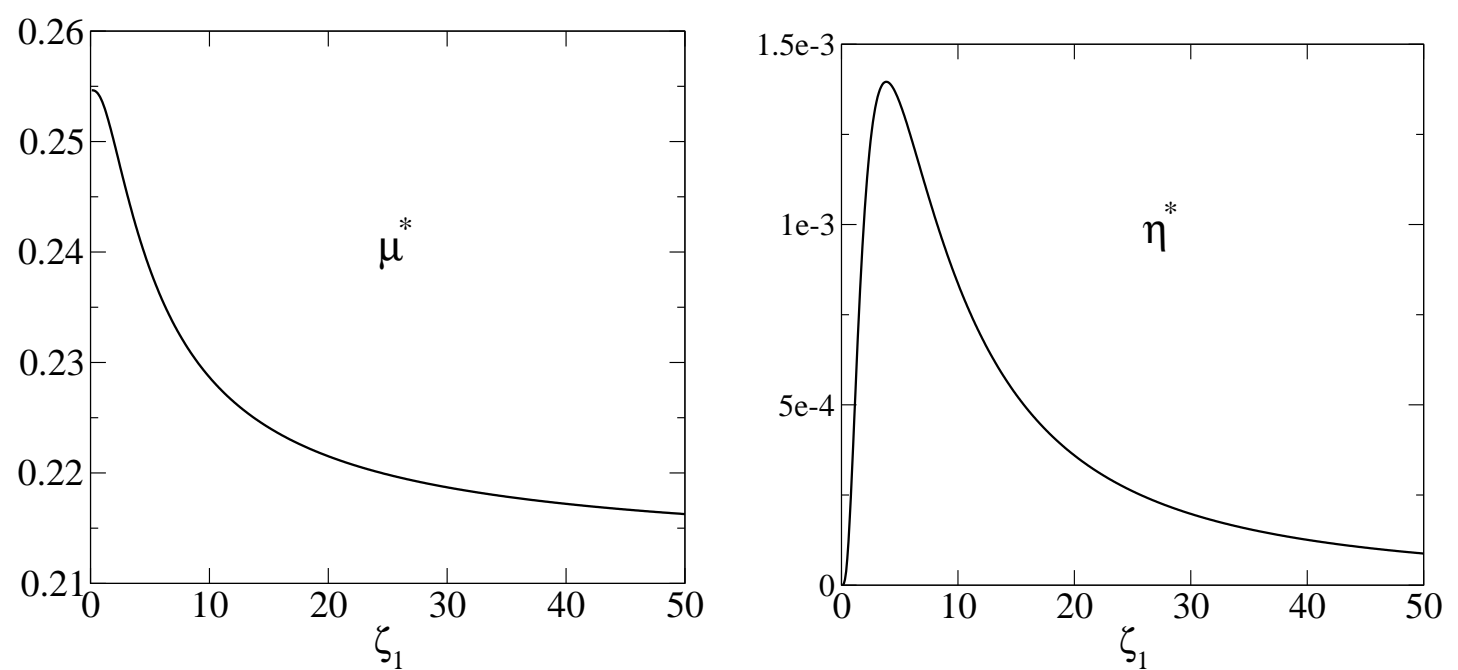

FIG. 3. Dimensionless coefficients as functions of $\zeta_{1}$ : shear viscosity $\mu^{*}$ (fig. 3a) and bulk viscosity $\eta^{*}$ (fig. 3b).

which represent small perturbations about an equilibrium state characterized by constant partial number densities $\mathrm{x}_{a} \mathrm{n}_{0}$, constant temperature $T_{0}$ and vanishing value of the velocity in the longitudinal direction parallel to the $x$ axis. Besides, the amplitudes of the perturbed fields - i.e., the overlined quantities - are small so that only linear deviations from equilibrium need to be take into account, while $\omega$ and $q$ are angular frequency and the wavenumber of the perturbations, respectively.

In the linear case, we can easily verify that the operators $\mathrm{D}$ and $\nabla^{\alpha}$ become $\mathrm{D}=\partial / \partial t$ and $\nabla^{\alpha}=\left(0,-\partial / \partial x^{i}\right)$. Hence, the insertion of expressions (8.1) and (8.2) into the field equations leads to following system of algebraic equations for the amplitude of the perturbations

$$
\left(\begin{array}{llll}
M_{11} & M_{12} & M_{13} & M_{14} \\
M_{21} & M_{22} & M_{23} & M_{24} \\
M_{31} & M_{32} & M_{33} & M_{34} \\
M_{41} & M_{42} & M_{43} & M_{44}
\end{array}\right)\left(\begin{array}{c}
\overline{\mathrm{n}}_{1} / \mathrm{n}_{0} \\
\overline{\mathrm{n}}_{2} / \mathrm{n}_{0} \\
\bar{v} / c \\
\bar{T} / T_{0}
\end{array}\right)=\left(\begin{array}{l}
0 \\
0 \\
0 \\
0
\end{array}\right)
$$

where the elements of the matrix are given by

$$
\begin{aligned}
& M_{11}=\omega_{*}+\imath\left[\mathrm{x}_{2}\left(1+\epsilon \alpha_{1} \mathrm{x}_{1}\right) \mathcal{D}_{12}^{*}-\frac{\mathcal{D}_{T}^{*}}{\mathrm{~h}_{*}}\right] q_{*}^{2}, \\
& M_{12}=-\imath\left[\mathrm{x}_{1}\left(1-\epsilon \alpha_{1} \mathrm{x}_{2}\right) \mathcal{D}_{12}^{*}+\frac{\mathcal{D}_{T}^{*}}{\mathrm{~h}_{*}}\right] q_{*}^{2}, \\
& M_{13}=-\mathrm{x}_{1} q_{*}, \quad M_{14}=\imath\left[\epsilon \alpha_{1} \mathrm{x}_{1} \mathrm{x}_{2} \mathcal{D}_{12}^{*}+\frac{\mathcal{D}_{T}^{*}}{\mathrm{~h}_{*}}\left(\mathrm{~h}_{*}-1\right)\right] q_{*}^{2}, \\
& M_{21}=-\imath\left[\mathrm{x}_{2}\left(1+\epsilon \alpha_{1} \mathrm{x}_{1}\right) \mathcal{D}_{12}^{*}-\frac{\mathcal{D}_{T}^{*}}{\mathrm{~h}_{*}}\right] q_{*}^{2}, \\
& M_{22}=\omega_{*}+\imath\left[\mathrm{x}_{1}\left(1-\epsilon \alpha_{1} \mathrm{x}_{2}\right) \mathcal{D}_{12}^{*}+\frac{\mathcal{D}_{T}^{*}}{\mathrm{~h}_{*}}\right] q_{*}^{2}, \quad M_{23}=-\mathrm{x}_{2} q_{*}, \\
& M_{24}=-M_{14}, \quad M_{31}=-q_{*}+\imath\left[\frac{\lambda^{*}}{\mathrm{~h}_{*}}-\mathrm{x}_{2}\left(1+\epsilon \alpha_{1} \mathrm{x}_{1}\right) \mathcal{D}^{*}\right] q_{*} \omega_{*}, \\
& M_{32}=-q_{*}+\imath\left[\frac{\lambda^{*}}{\mathrm{~h}_{*}}+\mathrm{x}_{1}\left(1-\epsilon \alpha_{1} \mathrm{x}_{2}\right) \mathcal{D}^{*}\right] q_{*} \omega_{*}, \\
& M_{33}=\mathrm{h}_{*} \omega_{*}+\imath\left(\eta^{*}+\frac{4}{3} \mu^{*}\right) q_{*}^{2},
\end{aligned}
$$




$$
\begin{aligned}
& M_{34}=-q_{*}-\imath\left[\frac{\lambda^{*}}{\mathrm{~h}_{*}}\left(\mathrm{~h}_{*}-1\right)+\epsilon \alpha_{1} \mathrm{x}_{1} \mathrm{x}_{2} \mathcal{D}^{*}\right] q_{*} \omega_{*}, \\
& M_{41}=\imath\left[\mathrm{x}_{2}\left(1+\epsilon \alpha_{1} \mathrm{x}_{1}\right) \mathcal{D}^{*}-\frac{\lambda^{*}}{\mathrm{~h}_{*}}\right] q_{*}^{2}, \\
& M_{42}=-\imath\left[\mathrm{x}_{1}\left(1-\epsilon \alpha_{1} \mathrm{x}_{2}\right) \mathcal{D}^{*}+\frac{\lambda^{*}}{\mathrm{~h}_{*}}\right] q_{*}^{2}, \quad M_{43}=-q_{*}, \\
& M_{44}=c_{v}^{*} \omega_{*}+\imath\left[\frac{\lambda^{*}}{\mathrm{~h}_{*}}\left(\mathrm{~h}_{*}-1\right)+\epsilon \alpha_{1} \mathrm{x}_{1} \mathrm{x}_{2} \mathcal{D}^{*}\right] q_{*}^{2} .
\end{aligned}
$$

In the above equations we have introduced a mean free time $\tau$ and starred quantities which correspond to dimensionless coefficients. They are given by

$$
\begin{gathered}
\tau=\frac{1}{n_{0} \mathcal{B}_{2}}, \quad q_{*}=q c \tau, \quad \omega_{*}=\omega \tau, \\
\mathrm{h}_{*}=\frac{\mathrm{h}}{k T}=\zeta_{1} G_{1}\left(1+\epsilon \alpha_{1} \times_{2}\right), \quad \alpha_{1}=\frac{G_{1}^{2} \zeta_{1}-\zeta_{1}-4 G_{1}}{G_{1}}, \\
c_{v}^{*}=\frac{c_{v}}{k}=\zeta_{1}^{2}+5 \zeta_{1} G_{1}-\zeta_{1}^{2} G_{1}^{2}-1+\epsilon \mathrm{x}_{2} \zeta_{1}\left[2 \zeta_{1}^{2} G_{1}\left(1-G_{1}^{2}\right)+\zeta_{1}\left(13 G_{1}^{2}-3\right)-20 G_{1}\right] .
\end{gathered}
$$

The system of algebraic equation (8.3) has a non-trivial solution if the determinant of the matrix of the coefficient vanishes. This condition leads to a dispersion relation which can be used to study the propagation of sound waves, as well as the dynamical behaviour of small disturbances induced by spontaneous internal fluctuations. For sound waves, the dispersion relation is solved by taking the angular frequency as a real input variable. As a result a wavenumber is found which in general is complex. In this case, the phase velocity $v_{p}$ and the attenuation coefficient $\alpha$ are defined by

$$
v_{p}=\frac{\omega}{\Re(q)} \quad \text { and } \quad \alpha=\Im(q) .
$$

On the other hand, for the eigenmodes, we consider the wavenumber as real input and solve the dispersion relation to obtain the complex angular frequency. The real part of the angular frequency $\Re(\omega)$ gives the oscillation frequency of a small internal perturbation with wavenumber $q$, while its imaginary part $\Im(\omega)$ describes the decay in time of the oscillation amplitude.

\section{A. Acoustic Solution in the Low-Frequency Limit}

In the low-frequency limit, the acoustic solution of the dispersion relation can be determined by expanding the reduced wavenumber $q_{*}$ in power series of the reduced oscillation frequency $\omega_{*}$ as

$$
q_{*}=a_{0} \omega_{*}+a_{1} \omega_{*}^{2}+\ldots,
$$

where $a_{0}, a_{1}, \ldots$ are complex expansion coefficients. By inserting the expansion (8.20) into the dispersion relation and equating equal powers of the reduced oscillation frequency $\omega_{*}$ we get

$$
\begin{aligned}
& a_{0}= \pm \sqrt{\frac{\mathbf{h}_{*}}{\gamma}} \\
& a_{1}=\imath \frac{a_{0}}{2 \gamma}\left\{\frac{4}{3} \mu^{*}+\eta^{*}+\frac{\left[(\gamma-1) a_{0}^{2}-1\right]^{2}}{a_{0}^{2}} \lambda^{*}+\gamma\left[(\gamma-1) a_{0}^{2}-1\right] \mathcal{D}^{*} \alpha_{1} \times_{1} \times_{2} \epsilon\right\},
\end{aligned}
$$

where $\gamma=\left(c_{v}^{*}+1\right) / c_{v}^{*}$ is the specific heat ratio.

The non-relativistic limit for the phase velocity and the attenuation coefficient in the low-frequency regime can be derived from the above expressions when we assume that the thermal energy of the gas mixture is smaller than the particle rest energy, i.e., when $\zeta_{1} \gg 1$. Hence, we have

$$
\begin{aligned}
& \frac{v_{p}}{v_{0}}= \pm\left\{1-\frac{7}{4 \zeta_{1}}\left(1-\mathrm{x}_{2} \epsilon\right)+\ldots\right\}, \\
& \frac{\alpha v_{0}}{\omega}= \pm \frac{7}{15 \pi}(\omega \tau)\left\{1+\frac{15}{28 \zeta_{1}}\left(1-\mathrm{x}_{2} \epsilon\right)+\ldots\right\},
\end{aligned}
$$


where $v_{0}$ denotes the adiabatic sound speed for a non-relativistic binary gas mixture:

$$
v_{0}=\sqrt{\frac{5}{3} \frac{k T_{0}}{m_{1}}\left(1-\mathrm{x}_{2} \epsilon\right)}
$$

At this point, it is important to mention that in the derivation of the above expressions for the phase velocity and the attenuation coefficient we have assumed that the masses of the particles of the constituents are not too disparate so that $\epsilon \ll 1$.

When $\zeta_{1} \rightarrow \infty$, we verify from expressions (8.23) and (8.24) that the phase velocity reduces to the adiabatic sound speed for a non-relativistic binary gas mixture, while the reduced attenuation coefficient $\alpha v_{0} / \omega$ varies linearly with the reduced frequency.

On the other hand, the ultra-relativistic limit for the phase velocity and the attenuation coefficient follows when $\zeta_{1} \ll 1$, i.e., when the thermal energy of the gas mixture is larger than the particle rest energy. In this case, we have

$$
\begin{aligned}
& \frac{v_{p}}{c}= \pm \frac{1}{\sqrt{3}}\left\{1-\frac{\left(1+2 \mathrm{x}_{2} \epsilon\right)}{24} \zeta_{1}^{2}+\ldots\right\} \\
& \frac{\alpha c}{\omega}= \pm \frac{2 \sqrt{3}}{5 \pi}(\omega \tau)\left\{1+\mathcal{O}\left(\zeta_{1}^{4}\right)\right\} .
\end{aligned}
$$

When $\zeta_{1} \rightarrow 0$ we note that the phase velocity is of order of the speed of light $v_{p}= \pm c / \sqrt{3}$, while the reduced attenuation coefficient, as in the non-relativistic limiting case, also varies linearly with the reduced oscillation frequency.

If we consider $\mathrm{x}_{2}=0$ in (8.23) - (8.27) we get the phase velocities and the attenuation coefficients for a single relativistic gas with Maxwellian particles. The expressions for the phase velocities (8.23) and (8.26) when $\mathrm{x}_{2}=0$ are the same as those in the work [27]. However, the attenuation coefficients (8.24) and (8.27) agree with those in [27] only in the first term without the corrections in the parameter $\zeta_{1}$. This fact can be easily understood, since here we are considering Maxwellian particles while in [27] a kind of hard-sphere potential was used. Due to the fact that the attenuation coefficient depends on the transport coefficients, which are functions on the type of potential, the corrections in the parameter $\zeta_{1}$ should not agree.

\section{B. Eigenmodes in the Small Wavenumber Limit}

By solving the dispersion relation to obtain the reduced oscillation frequency $\omega_{*}$ as a function of the reduced wavenumber $q_{*}$ we verify the existence of four longitudinal eigenmodes. Two of these hydrodynamic eigenmodes are sound modes describing sound propagation in opposite directions parallel to the wavenumber, while the other two eigenmodes are non-propagating modes that gives rise to purely diffusive effects. Expressions for these eigenmodes can be derived in the small wavenumber limit by expanding $\omega_{*}$ in power series of $q_{*}$ as

$$
\omega_{*}=a_{0}+a_{1} q_{*}+a_{2} q_{*}^{2}+\ldots
$$

By inserting expansion (8.28) into the dispersion relation and equating equal powers of $q_{*}$ we obtain for the sound modes:

$$
\begin{aligned}
& a_{0}=0, \quad a_{1}= \pm \sqrt{\frac{\gamma}{\mathrm{h}_{*}}}, \\
& a_{2}=-\imath \frac{a_{1}^{2}}{2 \gamma}\left\{\frac{4}{3} \mu^{*}+\eta^{*}+\frac{\left[a_{1}^{2}-(\gamma-1)\right]^{2}}{a_{1}^{2}} \lambda^{*}-\gamma \frac{\left[a_{1}^{2}-(\gamma-1)\right]}{a_{1}^{2}} \mathcal{D}^{*} \alpha_{1} \mathrm{x}_{1} \mathrm{x}_{2} \epsilon\right\},
\end{aligned}
$$

and for the diffusive modes:

$$
\begin{aligned}
& a_{0}=0, \quad \\
& a_{1}=-\frac{\imath}{2}\left\{\mathcal{D}_{12}^{*}+\frac{(\gamma-1)}{\gamma} \lambda^{*} \pm\left[\left(\mathcal{D}_{12}^{*}-\frac{(\gamma-1)}{\gamma} \lambda^{*}\right)^{2}+4 \frac{(\gamma-1)}{\gamma} \mathcal{D}^{*} \mathcal{D}_{T}^{*}\right]^{1 / 2}\right\} .
\end{aligned}
$$

As mentioned in Section VII the thermal-diffusion coefficient $\mathcal{D}_{T}$ and the diffusion-thermal coefficient $\mathcal{D}$ are of order $\epsilon$ so that the products $\mathcal{D}^{*} \epsilon$ in (8.30) and $\mathcal{D}^{*} \mathcal{D}_{T}^{*}$ in (8.32) can be neglected. In this case, the two sound modes can be written as

$$
\omega \tau= \pm \sqrt{\frac{\gamma}{\mathrm{h}_{*}}}(q c \tau)-\imath \frac{1}{2 \mathrm{~h}_{*}}\left\{\frac{4}{3} \mu^{*}+\eta^{*}+\frac{\left[\gamma-\mathrm{h}_{*}(\gamma-1)\right]^{2}}{\mathrm{~h}_{*} \gamma} \lambda^{*}\right\}(q c \tau)^{2}+\ldots
$$


and the two diffusive modes as

$$
\omega \tau=-\imath \frac{(\gamma-1)}{\gamma} \lambda^{*}(q c \tau)^{2}+\ldots \quad \text { and } \quad \omega \tau=-\imath \mathcal{D}_{12}^{*}(q c \tau)^{2}+\ldots
$$

We may observe from (8.34) a decoupling between entropy fluctuations and concentration fluctuations. A similar result is observed in a non-relavistic binary gas mixture of Maxwellian particles [28], where thermal-diffusion is zero and the non-propagating eigenmodes are related to thermal diffusivity and mass diffusion processes, respectively.

In the non-relativistic limit, the two sound modes read

$$
\omega \tau= \pm\left\{1-\frac{7}{4 \zeta_{1}}\left(1-\mathrm{x}_{2} \epsilon\right)+\ldots\right\}\left(q v_{0} \tau\right)-\imath \frac{7}{15 \pi}\left\{1-\frac{33}{7 \zeta_{1}}\left(1-\mathrm{x}_{2} \epsilon\right)+\ldots\right\}\left(q v_{0} \tau\right)^{2}+\ldots
$$

while the two diffusive modes become

$$
\begin{aligned}
& \omega \tau=-\imath \frac{3}{5 \pi}\left\{1-\frac{3}{2 \zeta_{1}}\left(1-\mathrm{x}_{2} \epsilon\right)+\ldots\right\}\left(q v_{0} \tau\right)^{2}+\ldots \\
& \omega \tau=-\imath \frac{3}{5 \pi} \mathcal{B}\left\{1-\frac{1}{2} \frac{(3+\mathcal{B})}{(2+\mathcal{B})}\left(1-2 \mathrm{x}_{2}\right) \epsilon\right. \\
& \left.-\frac{1}{2} \frac{(4+\mathcal{B})}{(2+\mathcal{B})}\left[1-\frac{26-36 \mathrm{x}_{2}+\left(13-14 \mathrm{x}_{2}\right) \mathcal{B}+\mathcal{B}^{2}}{2(2+\mathcal{B})(4+\mathcal{B})} \epsilon\right] \frac{1}{\zeta_{1}}+\ldots\right\}\left(q v_{0} \tau\right)^{2}+\ldots
\end{aligned}
$$

Above we have introduced the abbreviation $\mathcal{B}=\mathcal{B}_{2} / \mathcal{B}_{1}$.

The two sound modes in the ultra-relativistic limiting case are given by

$$
\begin{aligned}
\omega \tau & = \pm \frac{1}{\sqrt{3}}\left\{1-\frac{\left(1+2 \mathrm{x}_{2} \epsilon\right)}{24} \zeta_{1}^{2}+\ldots\right\}(q c \tau) \\
& -\imath \frac{2}{15 \pi}\left\{1-\frac{\left(1+2 \mathrm{x}_{2} \epsilon\right)}{8} \zeta_{1}^{2}+\ldots\right\}(q c \tau)^{2}+\ldots,
\end{aligned}
$$

and the two diffusive modes read

$$
\begin{aligned}
\omega \tau & =-\frac{\imath}{3 \pi}\left\{1-\frac{5}{24}\left(1+2 \mathrm{x}_{2} \epsilon\right) \zeta_{1}^{2}+\ldots\right\}(q c \tau)^{2}+\ldots \\
\omega \tau & =-\imath \frac{6 \mathcal{B}}{\pi} \frac{(2+\mathcal{B})}{(26+11 \mathcal{B})}\left\{1-\frac{2}{3} \frac{(11+5 \mathcal{B})}{(26+11 \mathcal{B})}[1\right. \\
& \left.\left.+\frac{\left(60-32 \mathrm{x}_{2}+\left(55-26 \mathrm{x}_{2}\right) \mathcal{B}+\left(13-6 \mathrm{x}_{2}\right) \mathcal{B}^{2}\right)}{2(2+\mathcal{B})(26+11 \mathcal{B})} \epsilon\right] \zeta_{1}^{2}+\ldots\right\}(q c \tau)^{2}+\ldots
\end{aligned}
$$

Note that fluctuations associated with the sound modes propagate with velocities $v=\Re(\omega) / q$ given by

$$
v= \pm v_{0}\left\{1-\frac{7}{4 \zeta_{1}}\left(1-\mathrm{x}_{2} \epsilon\right)+\ldots\right\}, \quad v= \pm \frac{c}{\sqrt{3}}\left\{1-\frac{1}{24}\left(1+2 \mathrm{x}_{2} \epsilon\right) \zeta_{1}^{2}+\ldots\right\}
$$

in the non-relativistic and in the ultra-relativistic limiting cases, respectively.

The expression (8.33) for the sound modes and the one for the diffusive mode (8.34a) are the same as those obtained in the work [29] for a single relativistic gas. If we set $x_{2}=0$ in (8.33) and in (8.34a) we get the sound and diffusive modes of a single relativistic gas with Maxwellian particles. As was pointed out in the last subsection the relativistic corrections in the parameter $\zeta_{1}$ should not agree with those in [29], since in the latter work a kind of hard-sphere potential was used.

The frequency and wavenumber regions of validity of the acoustic and eigenmodes solutions derived from the six field theory for a binary mixture can be determined by imposing, respectively, the conditions (see [29]):

$$
\frac{p}{\mu \omega}>1, \quad \text { and } \quad \frac{q \mu c}{p}<1,
$$

where $\mu$ is the shear viscosity of the mixture in the non-relativistic limiting case. The first condition tell us that an acoustic solution based on the classical hydrodynamic description is valid as long as the molecular collision frequency is larger than the wave frequency, while the second one tell us that the eigenmode solution is valid as long as the mean 
free path of the molecules is smaller than the wavelength of the internal fluctuations. In terms of the dimensionless frequency $\omega_{*}=\omega \tau$ and the dimensionless wavenumber $q_{*}=q c \tau$ we can rewrite the above conditions as

$$
\omega_{*}>\frac{3 \pi}{2}, \quad \text { and } \quad q_{*}<\frac{3 \pi}{2} .
$$

Finally, it is important to mention that another possible way to test the range of validity of an extended hydrodynamic description for the acoustic problem in relativistic gases is to consider how the maximum speed of propagation approaches the light speed with the increasing number of the moments. For the relativistic single gas case, a complete analysis based on this method was given in the work [30].

\section{CONCLUSIONS}

To sum up a mixture of $r$ constituents was analyzed within the framework of Boltzmann equation by using Grad's moment method. The mixture was described by $13 r+1$ basic fields of four-velocity, temperature of the mixture, particle number densities, diffusion fluxes, non-equilibrium pressures, heat fluxes and pressure deviator tensors. The $13 r+1$ field equations were obtained from Grad's distribution function applied to a mixture of Maxwellian particles where the rest masses of the constituents are not too disparate. From the system of $13 r+1$ field equations it was determined the constitutive equations for a binary mixture with six scalar fields of particle number densities, fourvelocity and temperature. The generalized laws of Fick, Fourier and Navier-Stokes were obtained and the transport coefficients of diffusion, thermal-diffusion, diffusion-thermal, thermal conductivity and bulk and shear viscosities were determined. Explicit expressions for these coefficients were given in the non-relativistic and ultra-relativistic limiting cases as well as their graphs in terms of a parameter which represents the ratio of the rest energy of a particle and the thermal energy of the gas mixture. An analysis of forced and free waves for the binary mixture was also performed. The phase velocity and attenuation coefficient were determined for the acoustic solution in the low frequency limit. It was also shown that there exist two sound modes describing sound propagation in opposite directions parallel to the wavenumber, and two non-propagating modes related to purely diffusive effects.

\section{ACKNOWLEDGMENTS}

This paper is dedicated to the memory of Dr. Leopoldo García-Colín Scherer. The work of G.M.K. has been supported by the Conselho Nacional de Desenvolvimento Científico e Tecnológico (Brazil).

\section{Appendix A: Evaluation of the production terms}

Let us evaluate the production term $\mathrm{P}_{a}^{\beta}$. By using the relationships (5.8) and the representation (5.13), the production term (2.11) becomes

$$
\begin{aligned}
\mathrm{P}_{a}^{\beta} & =\frac{c}{2} \sum_{b=1}^{r} \int\left(Q^{\prime \beta}-Q^{\beta}\right) f_{a} f_{b} F_{b a} \sigma_{a b} d \Omega \frac{d^{3} p_{b}}{p_{b 0}} \frac{d^{3} p_{a}}{p_{a 0}} \\
& =\frac{c}{2} \sum_{b=1}^{r} \int Q\left(\mathrm{k}^{\prime \beta}-\mathrm{k}^{\beta}\right) f_{a} f_{b} F_{b a} \sigma_{a b} d \Omega \frac{d^{3} p_{b}}{p_{b 0}} \frac{d^{3} p_{a}}{p_{a 0}} .
\end{aligned}
$$

If we write the element of solid angle as $d \Omega=\sin \Theta d \Theta d \Phi$ where $\Theta$ and $\Phi$ are the spherical angles of $\mathrm{k}^{\prime \beta}$ with respect to $\mathrm{k}^{\beta}$ and use the following result

$$
\int_{0}^{2 \pi} \int_{0}^{\pi}\left(\mathrm{k}^{\prime \beta}-\mathrm{k}^{\beta}\right) \sin \Theta d \Theta d \Phi=-2 \pi \int_{0}^{\pi} \mathrm{k}^{\beta}(1-\cos \Theta) \sin \Theta d \Theta
$$

the production term $(\mathrm{A} 1)$ reduces to

$$
\begin{array}{r}
\mathrm{P}_{a}^{\beta}=-\pi c \sum_{b=1}^{r} \int_{0}^{\pi} \int Q \mathrm{k}^{\beta} f_{a} f_{b} F_{b a} \sigma_{a b} d \Omega \frac{d^{3} p_{b}}{p_{b 0}} \frac{d^{3} p_{a}}{p_{a 0}}(1-\cos \Theta) \sin \Theta d \Theta \\
=-\pi c \sum_{b=1}^{r} \int_{0}^{\pi} \int\left[Q^{\beta}-\frac{\left(m_{a}^{2}-m_{b}^{2}\right) c^{2}}{P^{2}} P^{\beta}\right] f_{a} f_{b} F_{b a} \sigma_{a b} d \Omega \frac{d^{3} p_{b}}{p_{b 0}} \frac{d^{3} p_{a}}{p_{a 0}}(1-\cos \Theta) \sin \Theta d \Theta,
\end{array}
$$


thanks to (5.10). Now if we take into account (5.13) and transform the variables $\left(P^{\alpha}, Q^{\alpha}\right)$ into $\left(p_{a}^{\alpha}, p_{b}^{\alpha}\right)$ by using the relationships (5.6), we may perform the integrations of (A1) with respect to $\frac{d^{3} p_{b}}{p_{b 0}} \frac{d^{3} p_{a}}{p_{a 0}}$ and get

$$
\mathrm{P}_{a}^{\beta}=-\frac{\pi}{2 c^{2}} \mathcal{B}_{1} \sum_{b=1}^{r}\left[\left(T_{a}^{\beta \alpha}-T_{a \gamma}^{\gamma} \eta^{\beta \alpha}\right) N_{b \alpha}-\left(T_{b}^{\beta \alpha}-T_{b \gamma}^{\gamma} \eta^{\beta \alpha}\right) N_{a \alpha}\right]
$$

In the above expression $\mathcal{B}_{1}$ is the following integral when $n=1$ :

$$
\mathcal{B}_{n}=\int_{0}^{\pi} \mathcal{F}(\Theta)\left(1-\cos ^{n} \Theta\right) \sin \Theta d \Theta
$$

The same methodology may be used to determine the production term $\mathrm{P}_{a}^{\alpha \beta}$. First we obtain from (2.12)

$$
\begin{array}{r}
\mathrm{P}_{a}^{\alpha \beta}=\frac{c}{4} \sum_{b=1}^{r} \int\left[\left(Q^{\prime \alpha} Q^{\prime \beta}-Q^{\alpha} Q^{\beta}\right)+P^{\alpha}\left(Q^{\prime \beta}-Q^{\beta}\right)\right. \\
\left.+P^{\beta}\left(Q^{\prime \alpha}-Q^{\alpha}\right)\right] f_{a} f_{b} F_{b a} \sigma_{a b} d \Omega \frac{d^{3} p_{b}}{p_{b 0}} \frac{d^{3} p_{a}}{p_{a 0}} .
\end{array}
$$

Next by using the relationship

$$
\begin{aligned}
& \int_{0}^{2 \pi} \int_{0}^{\pi}\left(\mathrm{k}^{\prime \alpha} \mathrm{k}^{\prime \beta}-\mathrm{k}^{\alpha} \mathrm{k}^{\beta}\right) \sin \Theta d \Theta d \Phi \\
& =\pi \int_{0}^{\pi}\left(\frac{P^{\alpha} P^{\beta}}{P^{2}}-\eta^{\alpha \beta}-3 \mathrm{k}^{\alpha} \mathrm{k}^{\beta}\right)\left(1-\cos ^{2} \Theta\right) \sin \Theta d \Theta,
\end{aligned}
$$

and (A2) it follows that the production term (A6) reduces to

$$
\begin{aligned}
& \mathrm{P}_{a}^{\alpha \beta}=\frac{\pi c}{4} \sum_{b=1}^{r} \int_{0}^{\pi} \sin \Theta d \Theta \int\left\{\left[\frac{Q^{2} P^{\alpha} P^{\beta}}{P^{2}}-Q^{2} \eta^{\alpha \beta}-3 Q^{\alpha} Q^{\beta}+\frac{3\left(m_{a}^{2}-m_{b}^{2}\right) c^{2}}{P^{2}}\left(P^{\alpha} Q^{\beta}\right.\right.\right. \\
& \left.\left.\quad+P^{\beta} Q^{\alpha}\right)\right]\left(1-\cos ^{2} \Theta\right)-2\left[P^{\alpha} Q^{\beta}+P^{\beta} Q^{\alpha}-\frac{\left(m_{a}^{2}-m_{b}^{2}\right) c^{2}}{P^{2}}\left[P^{\beta}\left(P^{\alpha}-Q^{\alpha}\right)\right.\right. \\
& \left.\left.\left.\quad+P^{\alpha}\left(P^{\beta}-Q^{\beta}\right)\right]\right](1-\cos \Theta)\right\} f_{a} f_{b} F_{b a} \sigma_{a b} d \Omega \frac{d^{3} p_{b}}{p_{b 0}} \frac{d^{3} p_{a}}{p_{a 0}} .
\end{aligned}
$$

By performing the integration with respect to $\frac{d^{3} p_{b}}{p_{b 0}} \frac{d^{3} p_{a}}{p_{a 0}}$ leads to

$$
\begin{aligned}
& \mathrm{P}_{a}^{\alpha \beta}=-\frac{\pi}{2 c^{2}} \mathcal{B}_{1} \sum_{b=1}^{r}\left\{T_{a}^{\alpha \beta \gamma} N_{b \gamma}-T_{b}^{\alpha \beta \gamma} N_{a \gamma}-\frac{\left(m_{a}^{2}-m_{b}^{2}\right) c^{2}}{2}\left(N_{a}^{\alpha} N_{b}^{\beta}+N_{a}^{\beta} N_{b}^{\alpha}\right)+T_{a}^{\alpha \beta} T_{b \gamma}^{\gamma}\right. \\
& \left.-T_{b}^{\alpha \beta} T_{a \gamma}^{\gamma}\right\}+\frac{\pi}{8 c^{2}} \mathcal{B}_{2} \sum_{b=1}^{r}\left\{\left(\frac{m_{a}^{2}}{m_{b}^{2}}-5\right) T_{a}^{\alpha \beta} T_{b \gamma}^{\gamma}+\left(\frac{m_{b}^{2}}{m_{a}^{2}}-5\right) T_{b}^{\alpha \beta} T_{a \gamma}^{\gamma}+4\left(T_{a}^{\alpha \gamma} T_{b \gamma}^{\beta}\right.\right. \\
& \left.+T_{b}^{\alpha \gamma} T_{a \gamma}^{\beta}\right)-2\left(T_{a}^{\alpha \beta \gamma} N_{b \gamma}+T_{b}^{\alpha \beta \gamma} N_{a \gamma}\right)+\left(m_{a}^{2}+m_{b}^{2}\right) c^{2}\left(N_{a}^{\alpha} N_{b}^{\beta}+N_{a}^{\beta} N_{b}^{\alpha}\right) \\
& \left.-2 \eta^{\alpha \beta}\left[T_{a}^{\gamma \delta} T_{b \gamma \delta}-\left(\frac{m_{b}}{2 m_{a}}+\frac{m_{a}}{2 m_{b}}\right)^{2} T_{a \gamma}^{\gamma} T_{b \delta}^{\delta}\right]\right\} .
\end{aligned}
$$

It is worth to call attention that (A2) and (A7) result from the integration in the spherical angles, when the relative momentum four-vector after collision is expressed in terms of the one before collision. These are general expressions which are valid for all representations of the distribution function, like those that appear in the Chapman-Enskog and Grad methods.

\section{Appendix B: Linearized balance equations}

By introducing the operators $\mathrm{D}$ and $\nabla_{\alpha}$ defined by the relationship

$$
\mathrm{D}=U^{\alpha} \partial_{\alpha}, \quad \nabla_{\alpha}=\Delta^{\alpha \beta} \partial_{\beta}, \quad \text { so that } \quad \partial_{\alpha}=\frac{1}{c^{2}} U_{\alpha} \mathrm{D}+\nabla_{\alpha}, \quad \text { and } \quad U^{\alpha} \nabla_{\alpha}=0
$$


the linearized left-hand sides of (2.10) read

$$
\begin{aligned}
& \partial_{\alpha} N_{a}^{\alpha}=\mathrm{Dn}_{a}+\mathrm{n}_{a} \nabla_{\alpha} U^{\alpha}+\nabla_{\alpha} \mathrm{J}_{a}^{\alpha}, \\
& \partial_{\beta} T_{a}^{\alpha \beta}=\frac{\mathrm{n}_{a} \mathrm{~h}_{a}}{c^{2}} \mathrm{D} U^{\alpha}+\nabla_{\beta} \mathrm{p}_{a}^{\langle\alpha \beta}-\nabla^{\alpha}\left(\mathrm{p}_{a}+\varpi_{a}\right)+\frac{1}{c^{2}} \mathrm{D}\left(\mathrm{q}_{a}^{\alpha}+\mathrm{h}_{a} \mathrm{~J}_{a}^{\alpha}\right) \\
& \quad+\frac{U^{\alpha}}{c^{2}}\left[\mathrm{D}\left(\mathrm{n}_{a} \mathrm{e}_{a}\right)+\mathrm{n}_{a} \mathrm{~h}_{a} \nabla_{\beta} U^{\beta}+\nabla_{\beta}\left(\mathrm{q}_{a}^{\beta}+\mathrm{h}_{a} \mathrm{~J}_{a}^{\beta}\right)\right], \\
& \partial_{\gamma} T_{a}^{\alpha \beta \gamma}=U^{\alpha} U^{\beta}\left\{\frac{1}{3}\left[\left(m_{a}^{2}+2 C_{1 a}\right) \mathrm{Dn}_{a}-2 \zeta_{a} \mathrm{n}_{a} C_{1 a}^{\prime} \frac{\mathrm{D} T}{T}+2 C_{2 a} \mathrm{D} \varpi_{a}\right]+\mathrm{n}_{a} C_{1 a} \nabla_{\gamma} U^{\gamma}\right. \\
& \left.\quad-\frac{6 C_{3 a}}{c^{2}} \nabla_{\gamma} \mathrm{q}_{a}^{\gamma}+\frac{C_{6 a}}{c^{2}} \nabla_{\gamma} \mathrm{J}_{a}^{\gamma}\right\}+\frac{c^{2}}{6}\left[\left(m_{a}^{2}-C_{1 a}\right) \mathrm{D} \mathrm{n}_{a}+\zeta_{a} \mathrm{n}_{a} C_{1 a}^{\prime} \frac{\mathrm{D} T}{T}-C_{2 a} \mathrm{D} \varpi_{a}\right] \eta^{\alpha \beta} \\
& \quad+\frac{1}{6}\left(m_{a}^{2} \mathrm{n}_{a}+5 \mathrm{n}_{a} C_{1 a}\right)\left(U^{\alpha} \mathrm{D} U^{\beta}+U^{\beta} \mathrm{D} U^{\alpha}\right)+\frac{c^{2}}{6}\left[\left(m_{a}^{2}-C_{1 a}\right) \nabla_{\gamma} \mathrm{n}_{a}+\zeta_{a} \mathrm{n}_{a} C_{1 a}^{\prime} \frac{\nabla_{\gamma} T}{T}\right. \\
& \left.\quad-C_{2 a} \nabla_{\gamma} \varpi_{a}\right]\left(\eta^{\alpha \gamma} U^{\beta}+\eta^{\beta \gamma} U^{\alpha}\right)-\frac{5}{c^{2}} C_{3 a}\left(U^{\alpha} \mathrm{Dq}_{a}^{\beta}+U^{\beta} \mathrm{Dq}_{a}^{\alpha}\right)+C_{3 a}\left(\eta^{\alpha \beta} \nabla_{\gamma} \mathrm{q}_{a}^{\gamma}+\nabla^{\alpha} \mathrm{q}_{a}^{\beta}\right. \\
& \left.+\nabla^{\beta} q_{a}^{\alpha}\right)+\frac{c^{2}}{6}\left(m_{a}^{2} \mathrm{n}_{a}-\mathrm{n}_{a} C_{1 a}\right)\left(\eta^{\alpha \beta} \nabla_{\gamma} U^{\gamma}+\nabla^{\alpha} U^{\beta}+\nabla^{\beta} U^{\alpha}\right)+C_{4 a}\left(\mathrm{Dp}_{a}^{\langle\alpha \beta\rangle}+U^{\beta} \nabla_{\gamma} \mathrm{p}_{a}^{\langle\alpha \gamma\rangle}\right. \\
& \left.+U^{\alpha} \nabla_{\gamma} \mathrm{p}_{a}^{\langle\beta \gamma\rangle}\right)+C_{5 a}\left(\eta^{\alpha \beta} \nabla_{\gamma} \mathrm{J}_{a}^{\gamma}+\nabla^{\alpha} \mathrm{J}_{a}^{\beta}+\nabla^{\beta} \mathrm{J}_{a}^{\alpha}\right)+\frac{C_{5 a}+C_{6 a}}{c^{2}}\left(U^{\alpha} \mathrm{D} \mathrm{J}_{a}^{\beta}+U^{\beta} \mathrm{D}_{a}^{\alpha}\right)
\end{aligned}
$$

thanks to the representations (3.2), (3.6) and (5.1). Above we have introduced the notation $C_{1 a}^{\prime}=d C_{1 a}\left(\zeta_{a}\right) / d \zeta_{a}$.

Again by using (3.2), (3.6) and (5.1) we obtain from (A4) and (A9) the linearized production terms as functions of the $13 r+1$ moments of the distribution function:

$$
\begin{aligned}
& \mathrm{P}_{a}^{\beta}=-\frac{\pi}{2 c^{2}} \mathcal{B}_{1} \sum_{b=1}^{r}\left\{\left[\mathrm{n}_{b}\left(\mathrm{~h}_{a}+\mathrm{h}_{b}\right)-3 \mathrm{p}_{b}\right] \mathrm{J}_{a}^{\alpha}-\left[\mathrm{n}_{a}\left(\mathrm{~h}_{a}+\mathrm{h}_{b}\right)-3 \mathrm{p}_{a}\right] J_{b}^{\alpha}\right. \\
& \left.+\mathrm{n}_{b} \mathrm{q}_{a}^{\alpha}-\mathrm{n}_{a} \mathrm{q}_{b}^{\alpha}+3\left(\mathrm{n}_{b} \varpi_{a}-\mathrm{n}_{a} \varpi_{b}\right) U^{\alpha}\right\} \\
& \mathrm{P}_{a}^{\alpha \beta}=-\frac{\pi}{2 c^{2}} \mathcal{B}_{1} \sum_{b=1}^{r}\left\{\frac { c ^ { 2 } } { 3 } \left[\left(\mathrm{n}_{a} m_{a}^{2}+2 \mathrm{n}_{a} C_{1 a}+2 C_{2 a} \varpi_{a}\right) \mathrm{n}_{b}-\left(\mathrm{n}_{b} m_{b}^{2}+2 \mathrm{n}_{b} C_{1 b}\right.\right.\right. \\
& \left.\left.+2 C_{2 b} \varpi_{b}\right) \mathrm{n}_{a}\right] U^{\alpha} U^{\beta}+\frac{c^{4}}{6}\left[\left(\mathrm{n}_{a} m_{a}^{2}-\mathrm{n}_{a} C_{1 a}-C_{2 a} \varpi_{a}\right) \mathrm{n}_{b}-\left(\mathrm{n}_{b} m_{b}^{2}-\mathrm{n}_{b} C_{1 b}-C_{2 b} \varpi_{b}\right) \mathrm{n}_{a}\right] \eta^{\alpha \beta} \\
& -5\left[C_{3 a} \mathrm{n}_{b}\left(U^{\alpha} \mathbf{q}_{a}^{\beta}+U^{\beta} \mathbf{q}_{a}^{\alpha}\right)-C_{3 b} \mathrm{n}_{a}\left(U^{\alpha} \mathbf{q}_{b}^{\beta}+U^{\beta} \mathbf{q}_{b}^{\alpha}\right)\right]+c^{2}\left[C_{4 a} \mathrm{n}_{b} \mathrm{p}_{a}^{\langle\alpha \beta\rangle}-C_{4 b} \mathrm{n}_{a} \mathrm{p}_{b}^{\langle\alpha \beta\rangle}\right] \\
& +\mathrm{n}_{b}\left[C_{5 a}+C_{6 a}-\frac{c^{2}}{6}\left(m_{b}^{2}-C_{1 b}\right)\right]\left(U^{\alpha} \mathrm{J}_{a}^{\beta}+U^{\beta} \mathrm{J}_{a}^{\alpha}\right)-\mathrm{n}_{a}\left[C_{5 b}+C_{6 b}-\frac{c^{2}}{6}\left(m_{a}^{2}-C_{1 a}\right)\right]\left(U^{\alpha} \mathrm{J}_{b}^{\beta}\right. \\
& \left.+U^{\beta} \mathrm{J}_{b}^{\alpha}\right)+\left(\mathrm{n}_{b} \mathrm{e}_{b}-3 \mathrm{p}_{b}\right)\left[\mathrm{p}_{a}^{\langle\alpha \beta\rangle}-\left(\mathrm{p}_{a}+\varpi_{a}\right) \Delta^{\alpha \beta}+\frac{1}{c^{2}} U^{\beta}\left(\mathrm{q}_{a}^{\alpha}+\mathrm{h}_{a} \mathrm{~J}_{a}^{\alpha}\right)+\frac{1}{c^{2}} U^{\alpha}\left(\mathrm{q}_{a}^{\beta}+\mathrm{h}_{a} \mathrm{~J}_{a}^{\beta}\right)\right. \\
& \left.+\frac{\mathrm{n}_{a} \mathrm{e}_{a}}{c^{2}} U^{\alpha} U^{\beta}\right]-\left(\mathrm{n}_{a} \mathrm{e}_{a}-3 \mathrm{p}_{a}\right)\left[\mathrm{p}_{b}^{\langle\alpha \beta\rangle}-\left(\mathrm{p}_{b}+\varpi_{b}\right) \Delta^{\alpha \beta}+\frac{1}{c^{2}} U^{\beta}\left(\mathrm{q}_{b}^{\alpha}+\mathrm{h}_{b} \mathrm{~J}_{b}^{\alpha}\right)+\frac{1}{c^{2}} U^{\alpha}\left(\mathrm{q}_{b}^{\beta}\right.\right. \\
& \left.\left.+\mathrm{h}_{b} \mathrm{~J}_{b}^{\beta}\right)+\frac{\mathrm{n}_{b} \mathrm{e}_{b}}{c^{2}} U^{\alpha} U^{\beta}\right]-3 \varpi_{b}\left(\frac{\mathrm{n}_{a} \mathrm{e}_{a}}{c^{2}} U^{\alpha} U^{\beta}-\mathrm{p}_{a} \Delta^{\alpha \beta}\right)+3 \varpi_{a}\left(\frac{\mathrm{n}_{b} \mathrm{e}_{b}}{c^{2}} U^{\alpha} U^{\beta}-\mathrm{p}_{b} \Delta^{\alpha \beta}\right) \\
& \left.-\frac{\left(m_{a}^{2}-m_{b}^{2}\right) c^{2}}{2}\left[2 \mathrm{n}_{a} \mathrm{n}_{b} U^{\alpha} U^{\beta}+U^{\alpha}\left(\mathrm{n}_{a} \mathrm{~J}_{b}^{\beta}+\mathrm{n}_{b} \mathrm{~J}_{a}^{\beta}\right)+U^{\beta}\left(\mathrm{n}_{a} \mathrm{~J}_{b}^{\alpha}+\mathrm{n}_{b} \mathrm{~J}_{a}^{\alpha}\right)\right]\right\}
\end{aligned}
$$




$$
\begin{aligned}
& -\frac{\pi}{8 c^{2}} \mathcal{B}_{2} \sum_{b=1}^{r}\left\{\frac{2 c^{2}}{3}\left[\left(\mathrm{n}_{a} m_{a}^{2}+2 \mathrm{n}_{a} C_{1 a}+2 C_{2 a} \varpi_{a}\right) \mathrm{n}_{b}+\left(\mathrm{n}_{b} m_{b}^{2}+2 \mathrm{n}_{b} C_{1 b}+2 C_{2 b} \varpi_{b}\right) \mathrm{n}_{a}\right] U^{\alpha} U^{\beta}\right. \\
& +2 c^{2}\left[C_{4 a} \mathrm{n}_{b} \mathrm{p}_{a}^{\langle\alpha \beta\rangle}+C_{4 b} \mathrm{n}_{a} \mathrm{p}_{b}^{\langle\alpha \beta\rangle}\right]+\frac{c^{4}}{3}\left[\left(\mathrm{n}_{a} m_{a}^{2}-\mathrm{n}_{a} C_{1 a}-C_{2 a} \varpi_{a}\right) \mathrm{n}_{b}+\left(\mathrm{n}_{b} m_{b}^{2}-\mathrm{n}_{b} C_{1 b}\right.\right. \\
& \left.\left.-C_{2 b} \varpi_{b}\right) \mathrm{n}_{a}\right] \eta^{\alpha \beta}-10\left[C_{3 a} \mathrm{n}_{b}\left(U^{\alpha} \mathbf{q}_{a}^{\beta}+U^{\beta} \mathbf{q}_{a}^{\alpha}\right)+C_{3 b} \mathrm{n}_{a}\left(U^{\alpha} \mathrm{q}_{b}^{\beta}+U^{\beta} \mathbf{q}_{b}^{\alpha}\right)\right]+2 \mathrm{n}_{b}\left[C_{5 a}\right. \\
& \left.+C_{6 a}-\frac{c^{2}}{6}\left(m_{b}^{2}-C_{1 b}\right)\right]\left(U^{\alpha} \mathrm{J}_{a}^{\beta}+U^{\beta} \mathrm{J}_{a}^{\alpha}\right)+2 \mathrm{n}_{a}\left[C_{5 b}+C_{6 b}-\frac{c^{2}}{6}\left(m_{a}^{2}-C_{1 a}\right)\right]\left(U^{\alpha} \mathrm{J}_{b}^{\beta}\right. \\
& \left.+U^{\beta} \mathrm{J}_{b}^{\alpha}\right)+\left(5-\frac{m_{a}^{2}}{m_{b}^{2}}\right)\left[( \mathrm { n } _ { b } \mathrm { e } _ { b } - 3 \mathrm { p } _ { b } ) \left(\mathrm{p}_{a}^{\langle\alpha \beta\rangle}-\left(\mathrm{p}_{a}+\varpi_{a}\right) \Delta^{\alpha \beta}+\frac{1}{c^{2}} U^{\beta}\left(\mathrm{q}_{a}^{\alpha}+\mathrm{h}_{a} J_{a}^{\alpha}\right)\right.\right. \\
& \left.\left.+\frac{1}{c^{2}} U^{\alpha}\left(\mathrm{q}_{a}^{\beta}+\mathrm{h}_{a} \mathrm{~J}_{a}^{\beta}\right)+\frac{\mathrm{n}_{a} \mathrm{e}_{a}}{c^{2}} U^{\alpha} U^{\beta}\right)-3 \varpi_{b}\left(\frac{\mathrm{n}_{a} \mathrm{e}_{a}}{c^{2}} U^{\alpha} U^{\beta}-\mathrm{p}_{a} \Delta^{\alpha \beta}\right)\right]+\left(5-\frac{m_{b}^{2}}{m_{a}^{2}}\right)\left[\left(\mathrm{n}_{a} \mathrm{e}_{a}\right.\right. \\
& \left.-3 \mathrm{p}_{a}\right)\left(\mathrm{p}_{b}^{\langle\alpha \beta\rangle}-\left(\mathrm{p}_{b}+\varpi_{b}\right) \Delta^{\alpha \beta}+\frac{1}{c^{2}} U^{\beta}\left(\mathrm{q}_{b}^{\alpha}+\mathrm{h}_{b} \mathrm{~J}_{b}^{\alpha}\right)+\frac{1}{c^{2}} U^{\alpha}\left(\mathrm{q}_{b}^{\beta}+\mathrm{h}_{b} \mathrm{~J}_{b}^{\beta}\right)+\frac{\mathrm{n}_{b} \mathrm{e}_{b}}{c^{2}} U^{\alpha} U^{\beta}\right) \\
& \left.-3 \varpi_{a}\left(\frac{\mathrm{n}_{b} \mathrm{e}_{b}}{c^{2}} U^{\alpha} U^{\beta}-\mathrm{p}_{b} \Delta^{\alpha \beta}\right)\right]-\frac{8}{c^{2}} \mathrm{n}_{a} \mathrm{e}_{a} \mathrm{n}_{b} \mathrm{e}_{b} U^{\alpha} U^{\beta}+8 \mathrm{p}_{b} \mathrm{p}_{a}^{\langle\alpha \beta\rangle}+8 \mathrm{p}_{a} \mathrm{p}_{b}^{\langle\alpha \beta\rangle} \\
& -8\left(\mathrm{p}_{a} \mathrm{p}_{b}+\mathrm{p}_{a} \varpi_{b}+\mathrm{p}_{b} \varpi_{a}\right) \Delta^{\alpha \beta}-\frac{4}{c^{2}}\left[\left(\mathrm{n}_{a} \mathrm{e}_{a}-\mathrm{p}_{a}\right) U^{\beta}\left(\mathrm{q}_{b}^{\alpha}+\mathrm{h}_{b} \mathrm{~J}_{b}^{\alpha}\right)+\left(\mathrm{n}_{b} \mathrm{e}_{b}-\mathrm{p}_{b}\right) U^{\beta}\left(\mathrm{q}_{a}^{\alpha}+\mathrm{h}_{a} \mathrm{~J}_{a}^{\alpha}\right)\right. \\
& \left.+\left(\mathrm{n}_{a} \mathrm{e}_{a}-\mathrm{p}_{a}\right) U^{\alpha}\left(\mathrm{q}_{b}^{\beta}+\mathrm{h}_{b} \mathrm{~J}_{b}^{\beta}\right)+\left(\mathrm{n}_{b} \mathrm{e}_{b}-\mathrm{p}_{b}\right) U^{\alpha}\left(\mathrm{q}_{a}^{\beta}+\mathrm{h}_{a} \mathrm{~J}_{a}^{\beta}\right)\right]-\left(m_{a}^{2}+m_{b}^{2}\right) c^{2}\left[2 \mathrm{n}_{a} \mathrm{n}_{b} U^{\alpha} U^{\beta}\right. \\
& \left.+\mathrm{n}_{a} U^{\alpha} \mathrm{J}_{b}^{\beta}+\mathrm{n}_{b} U^{\beta} \mathrm{J}_{a}^{\alpha}+\mathrm{n}_{b} U^{\alpha} \mathrm{J}_{a}^{\beta}+\mathrm{n}_{a} U^{\beta} \mathrm{J}_{b}^{\alpha}\right]+2 \eta^{\alpha \beta}\left[3\left(\mathrm{p}_{a} \mathrm{p}_{b}+\mathrm{p}_{a} \varpi_{b}+\mathrm{p}_{b} \varpi_{a}\right)+\mathrm{n}_{a} \mathrm{e}_{a} \mathrm{n}_{b} \mathrm{e}_{\beta}\right] \\
& -2 \eta^{\alpha \beta}\left[( \frac { m _ { b } } { 2 m _ { a } } + \frac { m _ { a } } { 2 m _ { b } } ) ^ { 2 } \left(\mathrm{n}_{a} \mathrm{e}_{a} \mathrm{n}_{b} \mathrm{e}_{\beta}+9\left(\mathrm{p}_{a} \mathrm{p}_{b}+\mathrm{p}_{a} \varpi_{b}+\mathrm{p}_{b} \varpi_{a}\right)-3 \mathrm{n}_{a} \mathrm{e}_{a}\left(\mathrm{p}_{b}+\varpi_{b}\right)\right.\right. \\
& \left.\left.\left.-3 \mathrm{n}_{b} \mathrm{e}_{b}\left(\mathrm{p}_{a}+\varpi_{a}\right)\right)\right]\right\}
\end{aligned}
$$

\section{Appendix C: Maxwellian iteration procedure}

In the Maxwellian iteration procedure the equilibrium values of the constitutive quantities $\mathrm{p}_{1}^{\langle\alpha \beta\rangle}=\mathrm{p}_{2}^{\langle\alpha \beta\rangle}=0, \varpi_{1}=$ $\varpi_{2}=0, \mathrm{q}_{1}^{\alpha}=\mathrm{q}_{2}^{\alpha}=0$ and $\mathrm{J}_{1}^{\alpha}=-\mathrm{J}_{2}^{\alpha}=0$ are inserted on the left-hand sides of the equations $(6.6)-(6.9)$ and the first iterated values are obtained from the production terms, i.e., from the right-hand side of these equations.

First from (6.6) and (6.8) we obtain - by following the above mentioned methodology - the following algebraic system of equations for the determination of the diffusion $\mathrm{J}_{1}^{\alpha}$ and heat $\mathrm{q}_{1}^{\alpha}$, $\mathrm{q}_{2}^{\alpha}$ fluxes:

$$
\mathrm{d}_{1}^{\alpha}=\frac{\pi \mathcal{B}_{1}}{2 c^{2} k T}\left[\mathrm{n}\left(\mathrm{h}_{1}+\mathrm{h}_{2}-3 k T\right) \mathrm{J}_{1}^{\alpha}+\mathrm{n}_{2} \mathrm{q}_{1}^{\alpha}-n_{1} \mathrm{q}_{2}^{\alpha}\right]
$$




$$
\begin{aligned}
& m_{1} c^{2} G_{1} \mathrm{~d}_{1}^{\alpha}-\frac{m_{1}^{2} c^{4}}{k T^{2}} \mathrm{n}_{1}\left(G_{1}^{2}-5 \frac{G_{1}}{\zeta_{1}}-1\right) \nabla^{\alpha} \mathcal{T}=-\frac{\pi \mathcal{B}_{1}}{2 c^{2} k T}\left\{\mathrm{n}_{2}\left(5 c^{2} C_{31}-\mathrm{e}_{2}+3 k T\right) \mathrm{q}_{1}^{\alpha}\right. \\
& -\mathrm{n}_{1}\left(5 c^{2} C_{32}-\mathrm{e}_{1}+3 k T\right) \mathrm{q}_{2}^{\alpha}-c^{2}\left[\mathrm { n } _ { 1 } \left(C_{52}+C_{62}-\frac{\left(m_{1}^{2}-C_{11}\right) c^{2}}{6}+\frac{\mathrm{h}_{2}}{c^{2}}\left(\mathrm{e}_{1}-k T\right)\right.\right. \\
& \left.\left.\left.+\frac{\left(m_{1}^{2}-m_{2}^{2}\right) c^{2}}{2}\right)+\mathrm{n}_{2}\left(C_{51}+C_{61}-\frac{\left(m_{2}^{2}-C_{12}\right) c^{2}}{6}+\frac{\mathrm{h}_{1}}{c^{2}}\left(\mathrm{e}_{2}-k T\right)-\frac{\left(m_{1}^{2}-m_{2}^{2}\right) c^{2}}{2}\right)\right] \mathrm{J}_{1}^{\alpha}\right\} \\
& -\frac{\pi \mathcal{B}_{2}}{8 c^{2} k T}\left\{\left[2 \mathrm{n}_{1}\left(10 c^{2} C_{31}+8 k T\right)+\mathrm{n}_{2}\left(10 c^{2} C_{31}-\left(5-\frac{m_{1}^{2}}{m_{2}^{2}}\right)\left(\mathrm{e}_{2}-3 k T\right)+4\left(\mathrm{e}_{2}-k T\right)\right)\right] \mathrm{q}_{1}^{\alpha}\right. \\
& +\mathrm{n}_{1}\left[10 c^{2} C_{32}-\left(5-\frac{m_{2}^{2}}{m_{1}^{2}}\right)\left(\mathrm{e}_{1}-3 k T\right)+4\left(\mathrm{e}_{1}-k T\right)\right] \mathrm{q}_{2}^{\alpha}-2 c^{2}\left[2 \mathrm { n } _ { 1 } \left(C_{51}+C_{61}-4 k T \frac{\mathrm{h}_{1}}{c^{2}}\right.\right. \\
& \left.-\frac{\left(m_{1}^{2}-C_{11}\right) c^{2}}{6}-m_{1}^{2} c^{2}\right)+\mathrm{n}_{2}\left(C_{51}+C_{61}-\frac{\left(m_{2}^{2}-C_{12}\right) c^{2}}{6}+\left(5-\frac{m_{1}^{2}}{m_{2}^{2}}\right) \frac{\mathrm{e}_{2}-3 k T}{2} \frac{\mathrm{h}_{1}}{c^{2}}\right. \\
& \left.-2\left(\mathrm{e}_{2}-k T\right) \frac{\mathrm{h}_{1}}{c^{2}}-\frac{\left(m_{1}^{2}+m_{2}^{2}\right) c^{2}}{2}\right)-\mathrm{n}_{1}\left(C_{52}+C_{62}-\frac{\left(m_{1}^{2}-C_{11}\right) c^{2}}{6}-2\left(\mathrm{e}_{1}-k T\right) \frac{\mathrm{h}_{2}}{c^{2}}\right. \\
& \left.\left.\left.+\left(5-\frac{m_{2}^{2}}{m_{1}^{2}}\right) \frac{\mathrm{e}_{1}-3 k T}{2} \frac{\mathrm{h}_{2}}{c^{2}}-\frac{\left(m_{1}^{2}+m_{2}^{2}\right) c^{2}}{2}\right)\right] \mathrm{~J}_{1}^{\alpha}\right\}, \\
& m_{2} c^{2} G_{2} \mathrm{~d}_{1}^{\alpha}+\frac{m_{2}^{2} c^{4}}{k T^{2}} \mathrm{n}_{2}\left(G_{2}^{2}-5 \frac{G_{2}}{\zeta_{2}}-1\right) \nabla^{\alpha} \mathcal{T}=\frac{\pi \mathcal{B}_{1}}{2 c^{2} k T}\left\{\mathrm{n}_{1}\left(5 c^{2} C_{32}-\mathrm{e}_{1}+3 k T\right) \mathrm{q}_{2}^{\alpha}\right. \\
& -\mathrm{n}_{2}\left(5 c^{2} C_{31}-\mathrm{e}_{2}+3 k T\right) \mathrm{q}_{1}^{\alpha}+c^{2}\left[\mathrm { n } _ { 1 } \left(C_{52}+C_{62}-\frac{\left(m_{1}^{2}-C_{11}\right) c^{2}}{6}+\frac{\mathrm{h}_{2}}{c^{2}}\left(\mathrm{e}_{1}-k T\right)\right.\right. \\
& \left.\left.\left.+\frac{\left(m_{1}^{2}-m_{2}^{2}\right) c^{2}}{2}\right)+\mathrm{n}_{2}\left(C_{51}+C_{61}-\frac{\left(m_{2}^{2}-C_{12}\right) c^{2}}{6}+\frac{\mathrm{h}_{1}}{c^{2}}\left(\mathrm{e}_{2}-k T\right)-\frac{\left(m_{1}^{2}-m_{2}^{2}\right) c^{2}}{2}\right)\right] \mathrm{J}_{1}^{\alpha}\right\} \\
& +\frac{\pi \mathcal{B}_{2}}{8 c^{2} k T}\left\{\left[2 \mathrm{n}_{2}\left(10 c^{2} C_{32}+8 k T\right)+\mathrm{n}_{1}\left(10 c^{2} C_{32}+4\left(\mathrm{e}_{1}-k T\right)-\left(5-\frac{m_{2}^{2}}{m_{1}^{2}}\right)\left(\mathrm{e}_{1}-3 k T\right)\right)\right] \mathrm{q}_{2}^{\alpha}\right. \\
& +\mathrm{n}_{2}\left[10 c^{2} C_{31}+4\left(\mathrm{e}_{2}-k T\right)-\left(5-\frac{m_{1}^{2}}{m_{2}^{2}}\right)\left(\mathrm{e}_{2}-3 k T\right)\right] \mathrm{q}_{1}^{\alpha}+2 c^{2}\left[2 \mathrm { n } _ { 2 } \left(C_{52}+C_{62}-4 k T \frac{\mathrm{h}_{2}}{c^{2}}\right.\right. \\
& \left.-\frac{\left(m_{2}^{2}-C_{12}\right) c^{2}}{6}-m_{2}^{2} c^{2}\right)-\mathrm{n}_{2}\left(C_{51}+C_{61}-\frac{\left(m_{2}^{2}-C_{12}\right) c^{2}}{6}+\left(5-\frac{m_{1}^{2}}{m_{2}^{2}}\right) \frac{\mathrm{e}_{2}-3 k T}{2} \frac{\mathrm{h}_{1}}{c^{2}}\right. \\
& \left.-2\left(\mathrm{e}_{2}-k T\right) \frac{\mathrm{h}_{1}}{c^{2}}-\frac{\left(m_{1}^{2}+m_{2}^{2}\right) c^{2}}{2}\right)+\mathrm{n}_{1}\left(C_{52}+C_{62}-\frac{\left(m_{1}^{2}-C_{11}\right) c^{2}}{6}-2\left(\mathrm{e}_{1}-k T\right) \frac{\mathrm{h}_{2}}{c^{2}}\right. \\
& \left.\left.\left.+\left(5-\frac{m_{2}^{2}}{m_{1}^{2}}\right) \frac{\mathrm{e}_{1}-3 k T}{2} \frac{\mathrm{h}_{2}}{c^{2}}-\frac{\left(m_{1}^{2}+m_{2}^{2}\right) c^{2}}{2}\right)\right] \mathrm{~J}_{1}^{\alpha}\right\} .
\end{aligned}
$$

The solution of the above system of algebraic equations leads to the constitutive equations for $\mathrm{J}_{1}^{\alpha}, \mathrm{q}_{1}^{\alpha}$ and $\mathrm{q}_{2}^{\alpha}$ :

$$
\mathrm{J}_{1}^{\alpha}=-\mathrm{J}_{2}^{\alpha}=\mathcal{D}_{12} \mathrm{~d}_{1}^{\alpha}+\mathcal{D}_{T} \nabla^{\alpha} \mathcal{T}, \quad \mathrm{q}_{1}^{\alpha}=\lambda_{1} \nabla^{\alpha} \mathcal{T}+\mathcal{D}_{1} \mathrm{~d}_{1}^{\alpha}, \quad \mathrm{q}_{2}^{\alpha}=\lambda_{2} \nabla^{\alpha} \mathcal{T}+\mathcal{D}_{2} \mathrm{~d}_{1}^{\alpha},
$$

which are linear functions of the relativistic temperature gradient

$$
\nabla^{\alpha} \mathcal{T} \equiv \nabla^{\alpha} T-\frac{T}{\mathrm{nh}} \nabla^{\alpha} \mathrm{p}
$$

and of the generalized diffusion force

$$
\mathrm{d}_{1}^{\alpha} \equiv \frac{1}{k T}\left(\nabla^{\alpha} \mathrm{p}_{1}-\frac{\mathrm{n}_{1} \mathrm{~h}_{1}}{\mathrm{nh}} \nabla^{\alpha} \mathrm{p}\right), \quad \text { with } \quad \mathrm{d}_{2}^{\alpha}=-\mathrm{d}_{1}^{\alpha}
$$

Equation (C4a) represents generalized Fick's law, while (C4b,c) generalized Fourier's law. Here $\lambda_{1}, \lambda_{2}, \mathcal{D}_{12}, \mathcal{D}_{T}, \mathcal{D}_{1}$, $\mathcal{D}_{2}$ are scalar coefficients. 
The pressure deviator tensors $\mathrm{p}_{1}^{\langle\alpha \beta\rangle}, \mathrm{p}_{2}^{\langle\alpha \beta\rangle}$ are obtained from the algebraic system

$$
\begin{aligned}
& \frac{c^{2}}{3} \mathrm{n}_{1}\left(m_{1}^{2}-C_{11}\right) \nabla^{\langle\alpha} U^{\beta\rangle}=-\frac{\pi}{2 c^{2}} \mathcal{B}_{1}\left\{\mathrm{n}_{2}\left(c^{2} C_{41}+\mathrm{e}_{2}-3 k T\right) \mathrm{p}_{1}^{\langle\alpha \beta\rangle}-\mathrm{n}_{1}\left(c^{2} C_{42}+\mathrm{e}_{1}\right.\right. \\
& \left.-3 k T) \mathrm{p}_{2}^{\langle\alpha \beta\rangle}\right\}-\frac{\pi}{8 c^{2}} \mathcal{B}_{2}\left\{\left[2 \mathrm{n}_{1}\left(2 c^{2} C_{41}+4 \mathrm{e}_{1}-4 k T\right)+\mathrm{n}_{2}\left(2 c^{2} C_{41}+\left(5-\frac{m_{1}^{2}}{m_{2}^{2}}\right)\left(\mathrm{e}_{2}\right.\right.\right.\right. \\
& \left.-3 k T)+8 k T)] \mathrm{p}_{1}^{\langle\alpha \beta\rangle}+\mathrm{n}_{1}\left[2 c^{2} C_{42}+\left(5-\frac{m_{2}^{2}}{m_{1}^{2}}\right)\left(\mathrm{e}_{1}-3 k T\right)+8 k T\right] \mathrm{p}_{2}^{\langle\alpha \beta\rangle}\right\}, \\
& \frac{c^{2}}{3} \mathrm{n}_{2}\left(m_{2}^{2}-C_{12}\right) \nabla^{\langle\alpha} U^{\beta\rangle}=-\frac{\pi}{2 c^{2}} \mathcal{B}_{1}\left\{\mathrm{n}_{1}\left(c^{2} C_{42}+\mathrm{e}_{1}-3 k T\right) \mathrm{p}_{2}^{\langle\alpha \beta\rangle}-\mathrm{n}_{2}\left(c^{2} C_{41}+\mathrm{e}_{2}\right.\right. \\
& \left.-3 k T) \mathrm{p}_{1}^{\langle\alpha \beta\rangle}\right\}-\frac{\pi}{8 c^{2}} \mathcal{B}_{2}\left\{\left[2 \mathrm{n}_{2}\left(2 c^{2} C_{42}+4 \mathrm{e}_{2}-4 k T\right)+\mathrm{n}_{1}\left(2 c^{2} C_{42}+\left(5-\frac{m_{2}^{2}}{m_{1}^{2}}\right)\left(\mathrm{e}_{1}\right.\right.\right.\right. \\
& \left.-3 k T)+8 k T)] \mathrm{p}_{2}^{\langle\alpha \beta\rangle}+\mathrm{n}_{2}\left[2 c^{2} C_{41}+\left(5-\frac{m_{1}^{2}}{m_{2}^{2}}\right)\left(\mathrm{e}_{2}-3 k T\right)+8 k T\right] \mathrm{p}_{1}^{\langle\alpha \beta\rangle}\right\},
\end{aligned}
$$

that follow from (6.9) by using the Maxwellian iteration procedure. From the above system of algebraic equations we obtain the constitutive equations for the pressure deviator tensors as linear functions of the traceless part of the velocity gradient $\nabla^{\langle\alpha} U^{\beta\rangle}$, namely.

$$
\mathrm{p}_{1}^{\langle\alpha \beta\rangle}=2 \mu_{1} \nabla^{\langle\alpha} U^{\beta\rangle}, \quad \mathrm{p}_{2}^{\langle\alpha \beta\rangle}=2 \mu_{2} \nabla^{\langle\alpha} U^{\beta\rangle},
$$

where $\mu_{1}, \mu_{2}$ are scalar coefficients. The traceless part of the gradient of velocity is defined in the same way as the tensor $\Upsilon^{\alpha \beta}$ in (3.9).

Finally, the Maxwellian iteration procedure when applied to (6.7) leads to the following system of algebraic equations for the non-equilibrium pressures $\varpi_{1}, \varpi_{2}$ :

$$
\begin{aligned}
& \mathrm{n}_{1}\left[\frac{C_{11}-m_{1}^{2}}{3}+\frac{3 k m_{1}^{2}}{c_{v}}\left(G_{1}^{2}-6 \frac{G_{1}}{\zeta_{1}}-1\right)\right] \nabla_{\alpha} U^{\alpha}=-\frac{\pi}{4 c^{4}} \mathcal{B}_{1}\left\{\mathrm{n}_{2}\left(c^{4} C_{21}+6 \mathrm{e}_{2}\right) \varpi_{1}\right. \\
& \left.-\mathrm{n}_{1}\left(c^{4} C_{22}+6 \mathrm{e}_{1}\right) \varpi_{2}\right\}-\frac{\pi}{8 c^{4}} \mathcal{B}_{2}\left\{\frac { 3 \mathrm { n } _ { 1 } \mathrm { n } _ { 2 } } { 2 } \left[\left(m_{1}^{2}-m_{2}^{2}\right)\left(\mathrm{e}_{1} \mathrm{e}_{2}-3 k^{2} T^{2}\right)-3 k T\left(m_{1}^{2} \mathrm{e}_{1}-m_{2}^{2} \mathrm{e}_{2}\right)\right.\right. \\
& \left.-k T\left(m_{1}^{2} \mathrm{e}_{2}-m_{2}^{2} \mathrm{e}_{1}\right)\right] \frac{m_{2}^{2}-m_{1}^{2}}{m_{1}^{2} m_{2}^{2}}+2 \mathrm{n}_{1}\left[c^{4} C_{21}-6\left(\mathrm{e}_{1}+2 k T\right)\right] \varpi_{1}+\mathrm{n}_{2}\left[c^{4} C_{21}+6 k T\right. \\
& \left.-3\left(5-\frac{m_{2}^{2}}{m_{1}^{2}}\right) \mathrm{e}_{2}+6\left(\frac{m_{1}^{2}+m_{2}^{2}}{2 m_{1} m_{2}}\right)^{2}\left(\mathrm{e}_{2}-3 k T\right)\right] \varpi_{1}+\mathrm{n}_{1}\left[c^{4} C_{22}+6 k T-3\left(5-\frac{m_{1}^{2}}{m_{2}^{2}}\right) \mathrm{e}_{1}\right. \\
& \left.\left.+6\left(\frac{m_{1}^{2}+m_{2}^{2}}{2 m_{1} m_{2}}\right)^{2}\left(\mathrm{e}_{1}-3 k T\right)\right] \varpi_{2}\right\} \\
& \mathrm{n}_{2}\left[\frac{C_{12}-m_{2}^{2}}{3}+\frac{3 k m_{2}^{2}}{c_{v}}\left(G_{2}^{2}-6 \frac{G_{2}}{\zeta_{2}}-1\right)\right] \nabla_{\alpha} U^{\alpha}=-\frac{\pi}{4 c^{4}} \mathcal{B}_{1}\left\{\mathrm{n}_{1}\left(c^{4} C_{22}+6 \mathrm{e}_{1}\right) \varpi_{2}\right. \\
& \left.-\mathrm{n}_{2}\left(c^{4} C_{21}+6 \mathrm{e}_{2}\right) \varpi_{1}\right\}-\frac{\pi}{8 c^{4}} \mathcal{B}_{2}\left\{\frac { 3 \mathrm { n } _ { 1 } \mathrm { n } _ { 2 } } { 2 } \left[\left(m_{2}^{2}-m_{1}^{2}\right)\left(\mathrm{e}_{1} \mathrm{e}_{2}-3 k^{2} T^{2}\right)-3 k T\left(m_{2}^{2} \mathrm{e}_{2}-m_{1}^{2} \mathrm{e}_{1}\right)\right.\right. \\
& \left.-k T\left(m_{2}^{2} \mathrm{e}_{1}-m_{1}^{2} \mathrm{e}_{2}\right)\right] \frac{m_{1}^{2}-m_{2}^{2}}{m_{1}^{2} m_{2}^{2}}+2 \mathrm{n}_{2}\left[c^{4} C_{22}-6\left(\mathrm{e}_{2}+2 k T\right)\right] \varpi_{2}+\mathrm{n}_{2}\left[c^{4} C_{21}+6 k T\right. \\
& \left.-3\left(5-\frac{m_{2}^{2}}{m_{1}^{2}}\right) \mathrm{e}_{2}+6\left(\frac{m_{1}^{2}+m_{2}^{2}}{2 m_{1} m_{2}}\right)^{2}\left(\mathrm{e}_{2}-3 k T\right)\right] \varpi_{1}+\mathrm{n}_{1}\left[c^{4} C_{22}-3\left(5-\frac{m_{1}^{2}}{m_{2}^{2}}\right) \mathrm{e}_{1}+6 k T\right. \\
& \left.\left.+6\left(\frac{m_{1}^{2}+m_{2}^{2}}{2 m_{1} m_{2}}\right)^{2}\left(\mathrm{e}_{1}-3 k T\right)\right] \varpi_{2}\right\} \text {. }
\end{aligned}
$$

We note here that the above underlined terms are of order $\epsilon^{2}$ which must be neglected due to the hypothesis that the masses of the particles of the constituents are not too disparate, i.e., $m_{2}=m_{1}(1+\epsilon)$ with $\epsilon$ being a small quantity. 
Hence we obtain from $(\mathrm{C} 10)$ and (C11) the linear relationships between the non-equilibrium pressures and the velocity divergent $\nabla_{\alpha} U^{\alpha}$ :

$$
\varpi_{1}=-\eta_{1} \nabla_{\alpha} U^{\alpha}, \quad \varpi_{2}=-\eta_{2} \nabla_{\alpha} U^{\alpha}
$$

where $\eta_{1}, \eta_{2}$ are scalar coefficients.

Equations (C9) and (C12) represent the constitutive equations of a relativistic Newtonian fluid, also known as Navier-Stokes law.

\section{Appendix D: Generalized diffusion force and relativistic temperature gradient in the $\epsilon$-order}

In the $\epsilon$-order the generalized diffusion force and the relativistic temperature gradient read

$$
\begin{aligned}
\mathrm{d}_{1}^{\alpha}= & \mathrm{x}_{2} \nabla^{\alpha} \mathrm{n}_{1}-\mathrm{x}_{1} \nabla^{\alpha} \mathrm{n}_{2}+\epsilon \mathrm{x}_{1} \times_{2} \frac{G_{1}^{2} \zeta_{1}-\zeta_{1}-4 G_{1}}{G_{1}}\left[\nabla^{\alpha} \mathrm{n}_{1}+\nabla^{\alpha} \mathrm{n}_{2}+\frac{\mathrm{n}}{T} \nabla^{\alpha} T\right] \\
\nabla^{\alpha} \mathcal{T} & =\nabla^{\alpha} T-\frac{T}{\mathrm{nh}} \nabla^{\alpha} \mathrm{p}=\nabla^{\alpha} T-\frac{1}{\zeta_{1} G_{1}}\left(1-\epsilon \mathrm{x}_{2} \frac{G_{1}^{2} \zeta_{1}-\zeta_{1}-4 G_{1}}{G_{1}}\right) \\
& \times\left[\nabla^{\alpha} T+\frac{T}{\mathrm{n}} \nabla^{\alpha} \mathrm{n}_{1}+\frac{T}{\mathrm{n}} \nabla^{\alpha} \mathrm{n}_{2}\right]
\end{aligned}
$$

[1] F. Jüttner, "Das Maxwellsche Gesetz der Geschwindigkeitsverteilung in der Relativtheorie", Ann. Physik und Chemie 34, 856 (1911).

[2] F. Jüttner, "Die relativistische Quantentheorie des idealen Gases", Zeitschr. Physik 47, 542 (1928).

[3] A. Lichnerowicz and R. Marrot, "Propriétés statistiques des ensembles de particules en relativité restreite" C. R. Acad. Sci. Paris 210, 759 (1940).

[4] W. Israel, "Relativistic kinetic theory of a simple gas", J. Math. Phys. 4, 1163 (1963).

[5] D. C. Kelly, The kinetic theory of a relativistic gas, report (Miami University, Oxford, 1963).

[6] C. Marle, "Sur l'établissement des équations de l'hydrodynamique des fluides relativistes dissipatifs, I. L'équation de Boltzmann relativiste", Ann. Inst. Henri Poincarè X, 67 (1969); "II. Méthodes de résolution approchée de l'équation de Boltzmann relativiste", Ann. Inst. Henri Poincarè X, 127 (1969).

[7] J. M. Stewart, Non-equilibrium Relativistic Kinetic Theory, Lecture Notes in Physics, vol. 10 (Springer, Heidelberg, 1971).

[8] W. A. van Leeuwen, "On the relativistic kinetic theory of transport phenomena. I. General expressions for the transport coefficients", Proc. Kon. Ned. Akad. Wetensch. 74 B, 122 (1971) ; "II. The tensor, vector and scalar (pseudo-) eigenvalue equations of the collision operators for Maxwellian particles". Proc. Kon. Ned. Akad. Wetensch. 74 B, 134 (1971); "III. The solution of the linearized transport equations for Maxwellian particles", Proc. Kon. Ned. Akad. Wetensch. 74 B, 269 (1971); "IV. The calculation of the transport coefficients for Maxwellian particles", Proc. Kon. Ned. Akad. Wetensch. 74 B, 276 (1971) .

[9] W. T. Hermens, "Relativistic kinetic theory of chemical reactions and other transport phenomena. I: General discussion", Proc. Kon. Ned. Akad. Wetensch. 74 B, 376 (1971); "II. Normally reactive mixture", Proc. Kon. Ned. Akad. Wetensch. 74B, 461 (1971); "III. Highly reactive mixture", Proc. Kon. Ned. Akad. Wetensch. 74 B, 478 (1971).

[10] W. T. Hermens, W. A. van Leeuwen, C. G. van Weert and S. R. de Groot, "On relativistic kinetic gas theory. VIII. Reciprocal relations for a reactive mixture", Physica 60, 472 (1972).

[11] J. Guichelaar, "On relativistic kinetic gas theory. XIII. Sound propagation in a multi-component mixture", Physica 75, 593 (1974).

[12] W. A. van Leeuwen, A. J. Kox and S. R. de Groot, "On relativistic kinetic gas theory. XIV. Transport coefficients of a binary mixture. General expressions", Physica 79A, 233 (1975).

[13] A. J. Kox, W. A. van Leeuwen, and S. R. de Groot, "On relativistic kinetic gas theory. XVII. Diffusion and thermal diffusion in a binary mixture of hard spheres", Physica 84A, 165 (1976).

[14] A. J. Kox, "On relativistic kinetic gas theory. XVIII. Diffusion in a Lorentz gas of hard spheres", Physica 84A, 603 (1976).

[15] J. L. Anderson, "Variational principles for calculation of transport coefficients of relativistic multi-component systems", Physica A 87, 287 (1976).

[16] S. R. de Groot, W. A. van Leeuwen and Ch. G. van Weert, Relativistic Kinetic Theory (North-Holland, Amsterdam, 1980).

[17] C. Cercignani and G. M. Kremer, The Relativistic Boltzmann Equation: Theory and Applications (Birkhäuser, Basel, 2002).

[18] G. M. Kremer, "Note on the relativistic reaction rate coefficient", Physica A 380, 6165 (2007).

[19] P. H. Polak, W. A. van Leeuwen and S. R. de Groot, "On relativistic kinetic gas theory. X. Transport coefficients in the intermediate relativistic regime. Values for special models", Physica 66, 455 (1973).

[20] G. M. Kremer, "On the kinetic theory of relativistic gases", Continuum Mech. Thermodyn. 9, 13 (1997). 
[21] I-S. Liu, I. Müller and T. Ruggeri, "Relativistic thermodynamics of gases", Annals of Physics 169, 191 (1986).

[22] E. C. G. Stueckelberg and G. Wanders, "Thermodynamique en relativité générale", Helv. Phys. Acta 26, 417 (1953).

[23] H. Hebenstreit, "Balance equations for a relativistic plasma, I. Differential term", Physica 117 A, 631 (1983).

[24] G. M. Kremer and C. H. Patsko, "Relativistic ionized gases: Ohm and Fourier laws from Anderson and Witting model equation", Physica A 322, 329 (2003).

[25] S. Chapman and T. G. Cowling, The Mathematical Theory of Non-Uniform Gases, 3rd. edition (Cambridge University Press, Cambridge, 1970).

[26] G. M. Kremer, An Introduction to the Boltzmann Equation and Transport Processes in Gases (Springer, Berlin, 2010).

[27] C. Cercignani and G. M. Kremer, "Dispersion and absorption of plane harmonic waves in a relativistic gas", Continuum Mech. Thermodyn. 13, 171 (2001).

[28] J. P. Boon and S. Yip, Molecular Hydrodynamics (McGraw-Hill, New York, 1980).

[29] G. M. Kremer and W. Marques Jr., "Analysis of eigenmodes in a relativistic gas", Continuum Mech. Thermodyn. 24, 719 (2012).

[30] F. Brini and T. Ruggeri, "Maximum velocity for wave propagation in a relativistic rarefied gas", Continuum Mech. Thermodyn. 11, 331 (1999). 